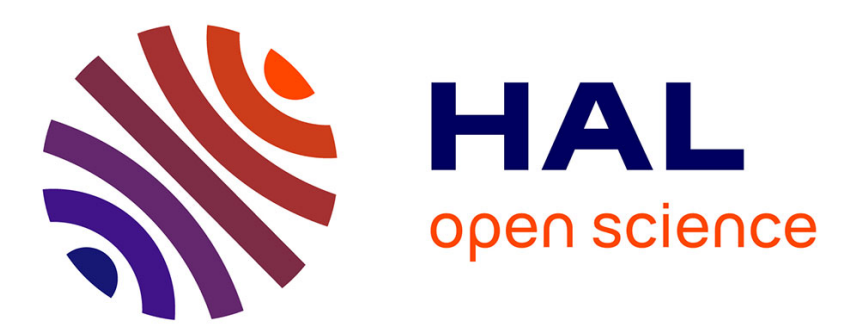

\title{
Remarks on the efficiency of POD for model reduction in non-linear dynamics of continuous elastic systems
}

R. Sampaio, Christian Soize

\section{To cite this version:}

R. Sampaio, Christian Soize. Remarks on the efficiency of POD for model reduction in non-linear dynamics of continuous elastic systems. International Journal for Numerical Methods in Engineering, 2007, 72 (1), pp.22-45. 10.1002/nme.1991 . hal-00686148

\section{HAL Id: hal-00686148 \\ https://hal.science/hal-00686148}

Submitted on 7 Apr 2012

HAL is a multi-disciplinary open access archive for the deposit and dissemination of scientific research documents, whether they are published or not. The documents may come from teaching and research institutions in France or abroad, or from public or private research centers.
L'archive ouverte pluridisciplinaire HAL, est destinée au dépôt et à la diffusion de documents scientifiques de niveau recherche, publiés ou non, émanant des établissements d'enseignement et de recherche français ou étrangers, des laboratoires publics ou privés. 


\title{
Remarks on the efficiency of POD for model reduction in nonlinear dynamics of continuous elastic systems
}

\author{
Rubens Sampaio ${ }^{*}$, Christian Soize ${ }^{2}$ \\ 1 PUC-Rio, Mechanical Engineering Department \\ Rua Marquês de São Vicente 225, Gávea \\ 22453-900, Rio de Janeiro, RJ, Brazil \\ 2 Universite de Marne la Vallee, Laboratoire de Mecanique \\ 5 Bd Descartes, 77454 Marne la Vallee, France
}

\section{SUMMARY}

The first objective of this paper is to analyze the efficiency of the reduced models constructed using the POD-basis and the LIN-basis in nonlinear dynamics for continuous elastic systems. The PODbasis is the Hilbertian basis constructed with the POD method while the LIN-basis is the Hilbertian basis derived from the generalized continuous eigenvalue problem associated with the underlying conservative part of the continuous elastic system and usually called the eigenmodes of vibration. The efficiency of the POD-basis or the LIN-basis is related to the rate of convergence in the frequency domain of the solution constructed with the reduced model with respect to its dimension. A basis will be more efficient than another if the reduced-order solution of the Galerkin projection converges to the solution of the dynamical system more rapidly than the reduced-order solution of the other.

\footnotetext{
*Correspondence to: PUC-Rio, Dept Eng Mecânica

Rua Marquês de São Vicente 225

22453-900, Rio de Janeiro, RJ, Brazil

Tel: +55213527 1172, Fax: +55213527 1165

rsampaio@mec.puc-rio.br

Contract/grant sponsor: This work was supported by the Brazilian Agency Conselho Nacional de Desenvolvimento Científico e Tecnológico (CNPq) and Faperj

Contract/grant sponsor: International Cooperation Project Capes-Cofecub; contract/grant number: $n^{\circ} 476 / 04$
} 
As a second objective of this paper, we present the usual results concerning the POD method using a continuous formulation, with respect to both time and space variables, and then deriving the numerical approximations. Such a presentation allows convergence discussions to be treated.

Six examples in nonlinear elastodynamics problems are presented in order to analyze the efficiency of the POD-basis and the LIN-basis. It is concluded that the POD-basis is not more efficient than the LIN-basis for the examples treated in nonlinear elastodynamics.

Copyright (c) 2006 John Wiley \& Sons, Ltd.

KEY WORDS: Reduced Model, Nonlinear Dynamics, Karhunen-Loève basis, POD, Snapshot

\section{Introduction}

We mean by POD-basis the Hilbertian basis constructed with the POD method. LINbasis means the Hilbertian basis derived from the generalized continuous eigenvalue problem associated with the underlying part of the continuous elastic system and usually called the eigenmodes of vibration.

In the last two decades, the number of papers dealing with Proper Orthogonal Decomposition (POD), also known as Karhunen-Loève basis (KL) [1, 2], to construct reduced models has increased a lot in diverse fields. It seems that the tendency is of further augmentation.

The prime objective of this paper is to compare the efficiency of the reduced model constructed with the POD-basis with the one constructed with the LIN-basis for nonlinear dynamics of continuous elastic systems, i. e. in nonlinear elastodynamics. We mean by efficiency of the POD-basis or the LIN-basis the rate of convergence in the frequency domain of the approximation constructed with the reduced model with respect to its dimension. In this paper we are not interested in constructing a reduced model adapted to a given excitation, which is generally the case if one uses the POD-basis. We are interested in constructing a reduced model as a predictive model for any excitation, which is the case if one uses the LINbasis. In linear and nonlinear elastodynamics it is usual to use the LIN-basis to construct the reduced model to predict the response to any excitation. Since the POD method seems to be 
an efficient tool to construct reduced models, this paper compares the efficiency of the two bases, LIN- and POD-basis, for nonlinear elastodynamics problems.

In general, the majority of the published papers presents the POD method in finite dimension, for time variable or for space or for both, directly writing the numerical approximations. Mathematical developments on the POD method in finite dimension for time variable and infinite dimension for space variable can be found, for instance, in $[3,4]$.

As a second objective of this paper, we present the usual results concerning the POD method using a continuous formulation, with respect to both time and space variables, and then deriving the numerical approximations. Such a presentation allows convergence discussions to be treated.

However, if one searches the literature for comparisons of the LIN-basis with the POD-basis, only a few cases can be found $[5,6]$ and for those the comparison is only made for a very small number of degrees of freedom and for discrete systems. For continuous systems the comparison is not generally made and in continuous nonlinear elastodynamics never. It is clear that if we want to know the dynamical response of a nonlinear continuous elastic system over a broadfrequency band, due to narrow- or broad-band excitation, such a comparison is necessary and it is not at all evident that the POD-basis is better than the LIN-basis for a given excitation. In addition one should remark that the POD-basis strongly depends on the excitation of the system while the LIN-basis does not depend and gives a reduced model valid for all excitations. Nevertheless in this paper the comparison of the efficiency of the two bases will be limited to the response to a given excitation.

We now try to separate the papers found in the vast literature in this field. The first class of papers deals with the discrete case and POD is seen as being equivalent to the Singular Value Decomposition or the Principal Component Analysis. This case is very different from the case of continuous systems because the dimension is finite. The second class of papers deals with the continuous case and the eigenvalue problem that one has to solve to compute the basis is effectively solved using the snapshot method (see, for instance $[3,4,5,6,7,8,9,10]$, for parabolic equations, for fluid dynamics problems, for nonlinear heat conduction problems, 
vibroimpact problems and for reconstruction of traveling waves). In general, in the two classes of papers, there is neither comparison of different bases of reduction nor of their efficiency in the reduction. In [6] a comparison is indeed made for a wind turbine, but the paper concerns low-frequency dynamics and the LIN-basis chosen seems to be not optimal.

We now describe the contents of the paper. Firstly, the weak formulation of the boundary value problem related to nonlinear elastodynamics is introduced in a functional analytic framework. Then the reduced model is presented introducing a Hilbertian basis constructed either with the POD-basis or the LIN-basis. Section 4 deals with the effective construction of the POD-basis introducing an adapted linear operator whose properties are mathematically studied. In Section 5 we present the finite element approximation of the problem. Sections 6 and 7 are devoted to the construction of the reduced model of the finite element approximation. Time discretization, error functions, and numerical solvers are presented in Section 8. A complete numerical study of the efficiency of the POD-basis with respect to the LIN-basis is given in Section 9 in which three types of nonlinear continuous elastodynamical systems are considered. Finally, we comment and summarize the main results obtained in Section 10.

\subsection{Geometry and boundarufonditionshe nonlinear boundary value problem}

We consider a damped elastic continuous medium occupying in its reference configuration an open bounded domain $\Omega$ of $\mathbb{R}^{d}$ with $d \leq 3$. The continuous nonlinear elastodynamical system is analyzed over the time interval $T=\mathbb{R}$. The smooth boundary $\partial \Omega$ of $\Omega$ is written as $\Gamma_{0} \cup \Gamma_{1}$. The displacement field in a configuration at time $t$ is defined on $\Omega$ with values in $\mathbb{R}^{n}$ and is denoted by $\mathbf{u}(\cdot, t)$. On $\Gamma_{0}$ there is a Dirichlet condition $\mathbf{u}(\cdot, t)=0$ for all $t$. On $\Gamma_{1}$ there is a given force field $\mathrm{g}^{s}(\cdot, t)$ depending on time $t$. In addition, there is a volumetric force field $\mathbf{g}^{v}(\cdot, t)$ applied in $\Omega$ and depending on time $t$. 


\subsection{Functional framework of the problem}

Before introducing the weak formulation of the boundary value problem we need a functional framework. We denote by $\langle$,$\rangle the Euclidean inner product in \mathbb{R}^{n}$ and by $\|\cdot\|$ its associated norm.

Let $\mathbb{H}=L^{2}\left(\Omega, \mathbb{R}^{n}\right)$ be the Hilbert space of all square integrable functions $\mathbf{x} \mapsto \mathbf{v}(\mathbf{x})$ from $\Omega$ into $\mathbb{R}^{n}$ equipped with the inner product $\langle\mathbf{v}, \delta \mathbf{v}\rangle_{\mathbb{H}}$ and the associated norm $\|\mathbf{v}\|_{\mathbb{H}}$ such that

$$
\langle\mathbf{v}, \delta \mathbf{v}\rangle_{\mathbb{H}}=\int_{\Omega}\langle\mathbf{v}(\mathbf{x}), \delta \mathbf{v}(\mathbf{x})\rangle \mathbf{d} \mathbf{x} \quad, \quad\|\mathbf{v}\|_{\mathbb{H}}^{2}=\int_{\Omega}\|\mathbf{v}(\mathbf{x})\|^{2} \mathbf{d x}
$$

The Hilbert space $\mathbb{V}$ of the admissible displacement fields is assumed to be a subspace of the Sobolev space $H^{\nu}\left(\Omega, \mathbb{R}^{n}\right)$ (with $\nu \geq 1$ ) constituted of functions $\mathbf{x} \mapsto \mathbf{v}(\mathbf{x})$ from $\Omega$ into $\mathbb{R}^{n}$ such that $\mathbf{v}=0$ on $\Gamma_{0}$. The Hilbert space $\mathbb{V}$ is equipped with the inner product $\langle\mathbf{v}, \delta \mathbf{v}\rangle_{\mathbb{V}}$ and the associated norm $\|\mathbf{v}\|_{\mathbb{V}}$. Let $\mathbb{V}^{\prime}$ be the continuous dual space of $\mathbb{V}$. We have $\mathbb{V} \subset \mathbb{H} \subset \mathbb{V}^{\prime}$ with continuous injections, $\mathbb{V}$ being dense in $\mathbb{H}$ and the injection from $\mathbb{V} \subset \mathbb{H}$ being compact. Let $\mathcal{H}=L^{2}(T, \mathbb{H})$ be the Hilbert space of square integrable functions $t \mapsto \mathbf{v}(\cdot, t)$ from $T$ into $\mathbb{H}$ equipped with inner product $\ll \mathbf{v}, \delta \mathbf{v} \gg_{\mathcal{H}}$ and the associated norm $\|\mathbf{v}\|_{\mathcal{H}}$ such that

$$
\ll \mathbf{v}, \delta \mathbf{v} \gg_{\mathcal{H}}=\int_{T}\langle\mathbf{v}(\cdot, t), \delta \mathbf{v}(\cdot, t)\rangle_{\mathbb{H}} d t \quad, \quad\|\mathbf{v}\|_{\mathcal{H}}^{2}=\int_{T}\|\mathbf{v}(\cdot, t)\|_{\mathbb{H}}^{2} d t
$$

Finally, let $\mathcal{V}=L^{2}(T, \mathbb{V})$ be the Hilbert subspace of $\mathcal{H}$ constituted of all the square integrable functions $t \mapsto \mathbf{v}(\cdot, t)$ from $T$ into $\mathbb{V}$ equipped with inner product $\ll \mathbf{v}, \delta \mathbf{v} \gg \mathcal{V}$ and the associated norm $\|\mathbf{v}\| \mathcal{v}$ such that

$$
\ll \mathbf{v}, \delta \mathbf{v} \gg_{\mathcal{V}}=\int_{T}\langle\mathbf{v}(\cdot, t), \delta \mathbf{v}(\cdot, t)\rangle_{\mathbb{V}} d t \quad, \quad\|\mathbf{v}\|_{\mathcal{V}}^{2}=\int_{T}\|\mathbf{v}(\cdot, t)\|_{\mathbb{V}}^{2} d t
$$

\subsection{Weak formulation of the problem}

For all $t$ fixed in $T$, let $\mathbf{v} \mapsto \ell(\mathbf{v}, t)$ be the linear form representing the applied forces and defined by

$$
\ell(\mathbf{v}, t)=\int_{\Omega}\left\langle\mathbf{g}^{v}(\mathbf{x}, t), \mathbf{v}(\mathbf{x})\right\rangle d \mathbf{x}+\int_{\Gamma_{1}}\left\langle\mathbf{g}^{s}(\mathbf{x}, t), \mathbf{v}(\mathbf{x})\right\rangle d s(\mathbf{x})
$$


It is assumed that the functions $\mathbf{g}^{v}$ and $\mathbf{g}^{s}$ are such that the linear form $\mathbf{v} \mapsto \ell(\mathbf{v}, t)$ is continuous on $\mathbb{V}$. The weak formulation $[11,12]$ of the problem is written as:

For a given $\ell$, find $\mathbf{u}$ in $\mathcal{V}$ such that, for all $t$ in $T$ and for all $\mathbf{v}$ in $\mathbb{V}$, we have

$$
m\left(\partial_{t}^{2} \mathbf{u}(t), \mathbf{v}\right)+d\left(\partial_{t} \mathbf{u}(t), \mathbf{v}\right)+k(\mathbf{u}(t), \mathbf{v})+k_{\mathrm{NL}}\left(\mathbf{u}(t), \partial_{t} \mathbf{u}(t), \mathbf{v}\right)=\ell(\mathbf{v}, t)
$$

In Eq. (5) the mappings $(\mathbf{w}, \mathbf{v}) \mapsto m(\mathbf{w}, \mathbf{v}),(\mathbf{w}, \mathbf{v}) \mapsto d(\mathbf{w}, \mathbf{v})$ and $(\mathbf{w}, \mathbf{v}) \mapsto k(\mathbf{w}, \mathbf{v})$ are assumed to be continuous, symmetric, and positive-definite bilinear forms on $\mathbb{V} \times \mathbb{V}$ with values in $\mathbb{R}$ corresponding to the mass, damping and the linear stiffness parts. For all $\mathbf{v}$ in $\mathbb{V}$, the mapping $(\mathbf{u}, \mathbf{w}) \mapsto k_{\mathrm{NL}}(\mathbf{u}, \mathbf{w}, \mathbf{v})$ is a nonlinear mapping on $\mathbb{V} \times \mathbb{H}$ with values in $\mathbb{R}$ corresponding to the nonlinear stiffness part. It is assumed that $k_{\mathrm{NL}}$ is such that Eq. (5) has a unique solution $\left(\mathbf{u}, \partial_{t} \mathbf{u}\right)$ in $\mathcal{V} \times \mathcal{H}$

3. Hilbertian basis for model reducing of the continuous elastodynamical system

\subsection{Principle of construction of the reduced model}

The principle of the construction of a reduced model consists (1) in constructing a Hilbertian basis $\left\{\mathbf{w}_{\alpha}, \alpha \geq 1\right\}$ of the Hilbert space $\mathbb{V},(2)$ in introducing the finite dimension subspace $\mathbb{V}_{N}$ of $\mathbb{V}$ such that $\mathbb{V}_{N}=\operatorname{span}\left\{\mathbf{w}_{1}, \ldots, \mathbf{w}_{N}\right\}$ and (3) in projecting Eq. (5) on $\mathbb{V}_{N}$. The solution $\mathbf{u}_{N}$ of the reduced model converges to the solution $\mathbf{u}$ in $\mathcal{V}$ of the problem when $N$ goes to infinity. Such a method is called the Galerkin method to get a reduced order model.

3.2. The LIN-basis as the Hilbertian basis derived from the generalized eigenvalue problem associated with the underlying linear part of the system

As explained in Section 1, we are interested in comparing the POD-basis with the LIN-basis, which has then to be constructed. From Eq. (5), an eigenvalue $\mu$ and the corresponding eigenmode $\mathbf{w}$ of the underlying linear part of the system are the solution of the following weak generalized eigenvalue problem,

$$
k(\mathbf{w}, \mathbf{v})=\mu m(\mathbf{w}, \mathbf{v}) \quad, \quad \forall \mathbf{v} \in \mathbb{V} .
$$


Since the bilinear forms $k$ and $m$ are continuous, symmetric and positive definite on $\mathbb{V} \times \mathbb{V}$, and since the injection from $\mathbb{V}$ into $\mathbb{H}$ is compact, then the set of the eigenvalues is a sequence of positive numbers $0\left\langle\mu_{1} \leq \mu_{2} \leq \ldots\right.$ and the set of the associated eigenfunctions $\left\{\mathbf{w}_{\alpha}, \alpha \geq 1\right\}$ is a Hilbertian basis of $\mathbb{V}$ satisfying the orthogonality properties

$$
k\left(\mathbf{w}_{\alpha}, \mathbf{w}_{\beta}\right)=\mu_{\alpha} \delta_{\alpha \beta} \quad, \quad m\left(\mathbf{w}_{\alpha}, \mathbf{w}_{\beta}\right)=\delta_{\alpha \beta},
$$

in which $\delta_{\alpha \beta}=0$ if $\alpha \neq \beta$ and $\delta_{\alpha \beta}=1$ if $\alpha=\beta$.

\subsection{The POD-basis as the Hilbertian basis constructed with the POD method}

The Hilbertian basis resulting from the POD method will be defined as the POD-basis.

We recall the Proper Orthogonal Decomposition (POD) in the deterministic case; the stochastic case is similar and corresponds to the Karhunen-Loève decomposition [13], although the two terminologies are used in the literature without discrimination if concerns the deterministic or stochastic case.

For a given function $\mathbf{u}$ in $\mathcal{V}=L^{2}(T, \mathbb{V})$, the POD method consists in finding the optimal Hilbertian basis $\left\{\mathbf{w}_{\alpha}, \alpha \geq 1\right\}$ of the Hilbert space $\mathbb{V}$ such that, for all fixed integer $N$, we have

$$
\left\|\mathbf{u}-\mathbf{u}_{N}\right\|_{\mathcal{V}} \leq\left\|\mathbf{u}-\widetilde{\mathbf{u}}_{N}\right\|_{\mathcal{V}}
$$

in which $\widetilde{\mathbf{u}}_{N}(\mathbf{x}, t)=\sum_{\alpha=1}^{N} \widetilde{q_{\alpha}}(t) \widetilde{\mathbf{w}}_{\alpha}(\mathbf{x})$, where $\left\{\widetilde{\mathbf{w}}_{\alpha}, \alpha \geq 1\right\}$ is any Hilbertian basis of $\mathbb{V}$ and, in which $\mathbf{u}_{N}(\mathbf{x}, t)=\sum_{\alpha=1}^{N} q_{\alpha}(t) \mathbf{w}_{\alpha}(\mathbf{x})$, where $\left\{\mathbf{w}_{\alpha}, \alpha \geq 1\right\}$ is the optimal basis of $\mathbb{V}$, solution of the optimal problem defined by Eq.(8). Since $\mathbf{u}$ is known, the functions $q_{\alpha}$ and $\widetilde{q}_{\alpha}$ are explicitly given by

$$
q_{\alpha}(t)=\left\langle\mathbf{u}(\cdot, t), \mathbf{w}_{\alpha}\right\rangle_{\mathbb{V}} \quad, \quad \widetilde{q}_{\alpha}(t)=\left\langle\mathbf{u}(\cdot, t), \widetilde{\mathbf{w}}_{\alpha}\right\rangle_{\mathbb{V}} .
$$

It is important to emphasize that the POD-basis is well adapted to represent a given function (in this case the function $\mathbf{u}$ ), but this does not mean that it is optimal for model reducing in the following sense. With the POD method, $\mathbf{u}$ and thus $\mathbf{u}_{N}$ depend on the excitation represented by the linear form $\ell$. Consequently, and this is well known, the optimal Hilbertian 
basis $\left\{\mathbf{w}_{\alpha}, \alpha \geq 1\right\}$ of $\mathbb{V}$ constructed by the POD method depends on $\ell$ and hence one should better write $\left\{\mathbf{w}_{\alpha}(\ell), \alpha \geq 1\right\}$. This means that if $\left\{\mathbf{w}_{\alpha}(\ell), \alpha \geq 1\right\}$ has been computed for a given $\ell$ it is not necessarily an optimal basis for a different $\ell$. That is, for each $\ell$ the basis has to be computed. In other words, such basis, computed for a given $\ell$ does not allow an efficient reduced model to be constructed for any excitation represented by a different linear form $\ell$. The constructed reduced model is then optimal for the excitation represented by $\ell$ for which the Hilbertian basis has been calculated. Consequently one can say that the reduced model constructed with the POD method is optimal for a given $\ell$ but not for another $\ell$, as it is well known.

For the linear case $\left(k_{\mathrm{NL}}=0\right)$, we then have three symmetric operators (mass, damping, stiffness operators). In this case, an optimal basis independent of $\ell$ can be constructed and consequentely allows an optimal reduced model to be constructed for any excitation represented by any linear form $\ell$ (see [14]). As proved in this paper, this optimal basis coincides with the linear basis (which does not depend on the excitation) when the damping of the system goes to zero.

4. Construction of the POD-basis for a continuous system

\subsection{Definition of a linear operator A for the POD method}

Let $\left(\mathbf{u}, \partial_{t} \mathbf{u}\right)$ be the unique solution in $\mathcal{V} \times \mathcal{H}$ of Eq. (5). Let $\left(\mathbf{x}, \mathbf{x}^{\prime}\right) \mapsto\left[a\left(\mathbf{x}, \mathbf{x}^{\prime}\right)\right]$ be the function from $\Omega \times \Omega$ into $\mathbb{R}^{n} \otimes \mathbb{R}^{n} \simeq \mathbb{M}_{n}(\mathbb{R})$ defined by

$$
\left[a\left(\mathbf{x}, \mathbf{x}^{\prime}\right)\right]=\int_{T} \mathbf{u}(\mathbf{x}, t) \otimes \mathbf{u}\left(\mathbf{x}^{\prime}, t\right) d t
$$

where $\mathbb{M}_{n}(\mathbb{R})$ is the set of all real $n \times n$ square matrices. Let $A$ be the linear integral operator from $\mathbb{H}$ in $\mathbb{H}$ defined by the kernel $\left[a\left(\mathbf{x}, \mathbf{x}^{\prime}\right)\right]$ such that

$$
\langle A \mathbf{w}, \delta \mathbf{w}\rangle_{\mathbb{H}}=\int_{\Omega} \int_{\Omega}\left\langle\left[a\left(\mathbf{x}, \mathbf{x}^{\prime}\right)\right] \mathbf{w}\left(\mathbf{x}^{\prime}\right), \delta \mathbf{w}(\mathbf{x})\right\rangle d \mathbf{x} d \mathbf{x}^{\prime}
$$

The operator $A$ is then, 


$$
(A \mathbf{w})(\mathbf{x})=\int_{\Omega}\left[\mathbf{a}\left(\mathbf{x}, \mathbf{x}^{\prime}\right)\right] \mathbf{w}\left(\mathbf{x}^{\prime}\right) \mathbf{d} \mathbf{x}^{\prime}
$$

\subsection{Properties of $A$}

We begin with a usual result, that describe the properties of a correlation operator, (Proposition 1), but we continue with Proposition 2 which proves that the eigenfunctions constitute a Hilbertian basis of $\mathbb{V}$ (less usual result). It should be noted that the Proposition 1 yields that the eigenfunctions of the eigenvalue problem defined by Eq. (13) is a Hilbertian basis in $\mathbb{H}$ but does not mean that a Hilbertian basis in $\mathbb{V}$ has been constructed. An additional element of proof is required to get such a result and is given in Proposition 2. This result is not currently found in the literature devoted to the POD method in infinite dimension for both time and space variables.

Proposition 1. For $\mathbf{u}$ in $\mathcal{V}$, the linear operator $A$ defined by Eq. (11) is real, symmetric, positive and is a Hilbert-Schmidt operator in $\mathbb{H}$.

Proof: First it can easily be verified that the operator $A$ is symmetric. Next to prove that the operator $A$ is a Hilbert-Schmidt operator [11], it is sufficient to show that $\int_{\Omega} \int_{\Omega}\left\|a\left(\mathbf{x}, \mathbf{x}^{\prime}\right)\right\|_{F}^{2} d \mathbf{x} d \mathbf{x}^{\prime}\left\langle\infty\right.$, in which $\left\|a\left(\mathbf{x}, \mathbf{x}^{\prime}\right)\right\|_{F}^{2}=\operatorname{tr}\left\{\left[a\left(\mathbf{x}, \mathbf{x}^{\prime}\right)\right]^{T}\left[a\left(\mathbf{x}, \mathbf{x}^{\prime}\right)\right]\right\}$ is the square of the Frobenius norm of the matrix $\left[a\left(\mathbf{x}, \mathbf{x}^{\prime}\right)\right]$, where tr denotes the trace of a matrix and the exponent $T$ means the transpose of a matrix. We have $\left\|a\left(\mathbf{x}, \mathbf{x}^{\prime}\right)\right\|_{F}^{2}=$ $\int_{T} \int_{T}\left\langle\mathbf{u}\left(\mathbf{x}^{\prime}, t\right), \mathbf{u}\left(\mathbf{x}^{\prime}, t^{\prime}\right)\right\rangle\left\langle\mathbf{u}(\mathbf{x}, t), \mathbf{u}\left(\mathbf{x}, t^{\prime}\right)\right\rangle d t d t^{\prime}$. Therefore

$$
\int_{\Omega} \int_{\Omega}\left\|a\left(\mathbf{x}, \mathbf{x}^{\prime}\right)\right\|_{F}^{2} d \mathbf{x} d \mathbf{x}^{\prime}=\int_{T} \int_{T}\left\langle\mathbf{u}(\cdot, t), \mathbf{u}\left(\cdot, t^{\prime}\right)\right\rangle_{\mathbb{H}}^{2} d t d t^{\prime}
$$

which proves that $A$ is a positive operator. We then have $\int_{\Omega} \int_{\Omega}\left\|a\left(\mathbf{x}, \mathbf{x}^{\prime}\right)\right\|_{F}^{2} d \mathbf{x} d \mathbf{x}^{\prime} \leq$ $\int_{T} \int_{T}\|\mathbf{u}(\cdot, t)\|_{\mathbb{H}}^{2}\left\|\mathbf{u}\left(\cdot, t^{\prime}\right)\right\|_{\mathbb{H}}^{2} d t d t^{\prime}$. Consequently,

$$
\int_{\Omega} \int_{\Omega}\left\|a\left(\mathbf{x}, \mathbf{x}^{\prime}\right)\right\|_{F}^{2} d \mathbf{x} d \mathbf{x}^{\prime} \leq\left(\int_{T}\|\mathbf{u}(\cdot, t)\|_{\mathbb{H}}^{2} d t\right)^{2}=\|u\|_{\mathcal{H}}^{4}\langle\infty
$$

which proves that $A$ is a Hilbert-Schmidt operator in $\mathbb{H}$. 
Proposition 2. Consider the eigenvalue problem

$$
A \mathbf{w}=\lambda \mathbf{w}
$$

related to the linear operator $A$. Then the set $\left\{\lambda_{\alpha}, \alpha \geq 1\right\}$ of the eigenvalues is a decreasing sequence of positive numbers such that $\sum_{\alpha=1}^{+\infty} \lambda_{\alpha}^{2}\left\langle+\infty\right.$ and the set $\left\{\mathbf{w}_{\alpha}, \alpha \geq 1\right\}$ of the associated eigenfunctions constitutes a Hilbertian basis of $\mathbb{V}$. The weak formulation of Eq. (13) is the following

$$
\langle A \mathbf{w}, \delta \mathbf{w}\rangle_{\mathbb{H}}=\lambda\langle\mathbf{w}, \delta \mathbf{w}\rangle_{\mathbb{H}} \quad, \quad \forall \delta \mathbf{w} \in \mathbb{H}
$$

Proof: Since $A$ is a symmetric positive Hilbert-Schmidt operator in $\mathbb{H}$ (see Proposition 1) we have the properties for the eigenvalues given in the proposition and $\left\{\mathbf{w}_{\alpha}, \alpha \geq 1\right\}$ is a Hilbertian basis of $\mathbb{H}$. We have now to show that $\left\{\mathbf{w}_{\alpha}, \alpha \geq 1\right\}$ is a Hilbertian basis of $\mathbb{V}$. Since $\mathbf{u}$ belongs to $\mathcal{V}$ then for all $t$ in $T, \mathbf{u}(\cdot, t)$ belongs to $\mathbb{V}$, and then $\mathbf{u}(\mathbf{x}, t)=0$ for all $\mathbf{x}$ in $\Gamma_{0}$. Consequently, Eqs. (10) and (11) show that for all $\mathbf{x}$ belonging to $\Gamma_{0}$, we have $A \mathbf{w}_{\alpha}(\mathbf{x})=\int_{\Omega}\left[a\left(\mathbf{x}, \mathbf{x}^{\prime}\right)\right] \mathbf{w}_{\alpha}\left(\mathbf{x}^{\prime}\right) d \mathbf{x}^{\prime}=0$. Since $A \mathbf{w}_{\alpha}=\lambda \mathbf{w}_{\alpha}$, we deduce that, for all $\alpha \geq 1$, $\mathbf{w}_{\alpha}=0$ on $\Gamma_{0}$. Therefore $\mathbf{w}_{\alpha}$ belongs to $\mathbb{V}$ if $\mathbf{w}_{\alpha}$ belongs to $H^{\nu}\left(\Omega, \mathbb{R}^{n}\right)$. From reference [15], we have this property if $\int_{\Omega} \int_{\Omega}\left\|\partial^{2 \nu} a\left(\mathbf{x}, \mathbf{x}^{\prime}\right) / \partial x_{\ell}^{\nu} \partial{x^{\prime}}_{j}\right\|_{F}^{2} d \mathbf{x} d \mathbf{x}^{\prime}\langle+\infty$. We have

$$
\begin{aligned}
\int_{\Omega} \int_{\Omega}\left\|\frac{\partial^{2 \nu} a\left(\mathbf{x}, \mathbf{x}^{\prime}\right)}{\partial x_{\ell}^{\nu} \partial x_{j}^{\prime \nu}}\right\|_{F}^{2} d \mathbf{x} d \mathbf{x}^{\prime}= & \\
& \int_{T} \int_{T}\left\langle\partial_{\ell}^{\nu} \mathbf{u}(\cdot, t), \partial_{\ell}^{\nu} \mathbf{u}\left(\cdot, t^{\prime}\right)\right\rangle_{\mathbb{H}}\left\langle\partial_{j}^{\nu} \mathbf{u}(\cdot, t), \partial_{j}^{\nu} \mathbf{u}\left(\cdot, t^{\prime}\right)\right\rangle_{\mathbb{H}} d t d t^{\prime},
\end{aligned}
$$

in which $\partial_{j}=\partial / \partial x_{j}$. Therefore

$$
\begin{aligned}
& \int_{\Omega} \int_{\Omega}\left\|\frac{\partial^{2 \nu} a\left(\mathbf{x}, \mathbf{x}^{\prime}\right)}{\partial x_{\ell}^{\nu} \partial x_{j}^{\prime \nu}}\right\|_{F}^{2} d \mathbf{x} d \mathbf{x}^{\prime} \\
& \quad \leq \int_{T}\left\|\partial_{\ell}^{\nu} \mathbf{u}(\cdot, t)\right\|_{\mathbb{H}}\left\|\partial_{j}^{\nu} \mathbf{u}(\cdot, t)\right\|_{\mathbb{H}} d t \int_{T}\left\|\partial_{\ell}^{\nu} \mathbf{u}\left(\cdot, t^{\prime}\right)\right\|_{\mathbb{H}}\left\|\partial_{j}^{\nu} \mathbf{u}\left(\cdot, t^{\prime}\right)\right\|_{\mathbb{H}} d t^{\prime} \\
& \leq\left\|\partial_{\ell}^{\nu} \mathbf{u}\right\|_{\mathcal{H}}^{2}\left\|\partial_{j}^{\nu} \mathbf{u}\right\|_{\mathcal{H}}^{2}\langle+\infty
\end{aligned}
$$

because $\mathbf{u}$ belongs to $\mathcal{V} \subset L^{2}\left(T, H^{\nu}\left(\Omega, \mathbb{R}^{n}\right)\right)$, that completes the proof. 
5. Finite element approximation

In this section we present the finite element approximation of the weak formulation presented in Section 2 and the finite element approximation of the LIN-basis and the POD-basis presented in Sections 3 and 4.

\subsection{Finite element approximation of the weak formulation of the problem}

The finite element approximation of Eq. (5) with $p$ degrees of freedom introduces the approximation of $\mathbf{u}(\cdot, t) \in \mathbb{V}$ by $\mathbf{u}(\mathbf{x}, t) \simeq[B(\mathbf{x})] \mathbf{y}(t)$ in which $\mathbf{y}(t)$ is a $\mathbb{R}^{p}$-vector of the degrees of freedom and the matrix $[B(\mathbf{x})]$ is constructed with the interpolation functions of the finite elements $[11,16]$. We then obtain the following matrix equation in $\mathbb{R}^{p}$,

$$
[\mathbb{M}] \ddot{\mathbf{y}}(t)+[\mathbb{D}] \dot{\mathbf{y}}(t)+[\mathbb{K}] \mathbf{y}(t)+\mathbf{f}_{\mathrm{NL}}(\mathbf{y}(t), \dot{\mathbf{y}}(t))=\mathbf{f}(t)
$$

where $[\mathbb{M}],[\mathbb{D}],[\mathbb{K}]$ and $\mathbf{f}_{\mathrm{NL}}(\mathbf{y}(t), \dot{\mathbf{y}}(t))$ are the mass, damping, stiffness matrices and the nonlinear vector forces corresponding to the finite element discretization of the bilinear forms $m, d, k$ and the nonlinear mapping $k_{\mathrm{NL}}\left(\mathbf{u}(t), \partial_{t} \mathbf{u}(t), \mathbf{v}\right)$. The applied vector load $\mathbf{f}(t)$ corresponds to the finite element discretization of the linear form $\ell(\mathbf{v}, t)$. This vector load $\mathbf{f}(t)$ is assumed to be written as

$$
\mathbf{f}(t)=a g(t) \mathbf{f}_{0} \quad,
$$

in which $a$ is the amplitude and $\mathbf{f}_{0}$ is a normalized vector describing the spatial distribution of the applied forces. The impulse $t \mapsto g(t)$ is a square integrable real-valued function on $\mathbb{R}$ whose Fourier Transform $\omega \mapsto \widehat{g}(\omega)=\int_{\mathbb{R}} e^{-i \omega t} g(t) d t$ has a bounded support $\underline{B}_{e} \cup B_{e}$ with

$$
B_{e}=\left[\Omega_{c}-\Delta \Omega / 2, \Omega_{c}+\Delta \Omega / 2\right] \quad, \quad \underline{B}_{e}=\left[-\Omega_{c}-\Delta \Omega / 2,-\Omega_{c}+\Delta \Omega / 2\right] \quad .
$$

In addition it is assumed that $\max _{\omega \in B}|\widehat{g}(\omega)|=1$. 
5.2. Construction of the LIN-basis: finite element approximation for the eigenmodes of the underlying linear part of the system

The finite element approximation of Eq. (6) can directely be deduced from Eq. (16). The eigenvalues (the square of the eigenfrequencies)

$$
0<\widehat{\mu}_{1} \leq \widehat{\mu}_{2} \leq \ldots \leq \widehat{\mu}_{p}
$$

and the associated eigenmodes $\mathbf{z}_{1}, \mathbf{z}_{2}, \ldots, \mathbf{z}_{p}$ are the solution of the following generalized eigenvalue problem

$$
[\mathbb{K}] \mathbf{z}=\widehat{\mu}[\mathbb{M}] \mathbf{z}
$$

with the orthogonality properties

$$
\left\langle[\mathbb{K}] \mathbf{z}_{\alpha}, \mathbf{z}_{\beta}\right\rangle=\widehat{\mu}_{\alpha} \delta_{\alpha \beta} \quad, \quad\left\langle[\mathbb{M}] \mathbf{z}_{\alpha}, \mathbf{z}_{\beta}\right\rangle=\delta_{\alpha \beta} .
$$

\subsection{Construction of the POD-basis: finite element approximation of the POD method}

The finite element mesh and the finite elements used for the discretization of the POD method are the same as the finite element discretization of the weak formulation of the problem. Consequently, we have $\mathbf{u}(\mathbf{x}, t) \simeq[B(\mathbf{x})] \mathbf{y}(t)$ and $\mathbf{w}(\mathbf{x}) \simeq[B(\mathbf{x})] \mathbf{z}$. The finite element discretization of Eq. (13) is written as

$$
[A] \mathbf{z}=\widehat{\lambda}[H] \mathbf{z}
$$

in which $[A]$ is a positive symmetric $p \times p$ real matrix given by

$$
[A]=\int_{T}[H] \mathbf{y}(t)([H] \mathbf{y}(t))^{T} d t,
$$

and $[H]$ is a positive-definite symmetric $p \times p$ real matrix given by

$$
[H]=\int_{\Omega}[B(\mathbf{x})]^{T}[B(\mathbf{x})] d \mathbf{x} .
$$

The eigenvalues are positive numbers such that

$$
\widehat{\lambda}_{1} \geq \widehat{\lambda}_{2} \geq \ldots \widehat{\lambda}_{p} \geq 0
$$

The associated eigenvectors $\mathbf{z}_{1}, \mathbf{z}_{2}, \ldots, \mathbf{z}_{p}$ satisfy the orthogonality properties

$$
\left\langle[A] \mathbf{z}_{\alpha}, \mathbf{z}_{\beta}\right\rangle=\widehat{\lambda}_{\alpha} \delta_{\alpha \beta} \quad, \quad\left\langle[H] \mathbf{z}_{\alpha}, \mathbf{z}_{\beta}\right\rangle=\delta_{\alpha \beta}
$$


6. Reduced model of the finite element approximation

Let $\left\{\mathbf{z}_{1}, \ldots, \mathbf{z}_{p}\right\}$ be an algebraic basis of $\mathbb{R}^{p}$. Such a basis can be either the LIN-basis constructed in Section 5.2 or the POD-basis constructed in Section 5.3. The reduced model in the finite element approximation is obtained by projection of Eq. (16) on the subspace $V_{N}$ of $\mathbb{R}^{p}$ spanned by $\left\{\mathbf{z}_{1}, \ldots, \mathbf{z}_{N}\right\}$ with $N \ll p$. Let $\left[Z_{N}\right]$ be the $(p \times N)$ real matrix whose columns are the vectors $\left\{\mathbf{z}_{1}, \ldots, \mathbf{z}_{N}\right\}$. The generalized applied force $\mathbf{F}^{N}(t)$ is a $\mathbb{R}^{N}$. vector such that $\mathbf{F}^{N}(t)=\left[Z_{N}\right]^{T} \mathbf{f}(t)$. The generalized mass, damping and stiffness matrices $\left[M_{N}\right]=\left[Z_{N}\right]^{T}[\mathbb{M}]\left[Z_{N}\right],\left[D_{N}\right]=\left[Z_{N}\right]^{T}[\mathbb{D}]\left[Z_{N}\right]$ and $\left[K_{N}\right]=\left[Z_{N}\right]^{T}[\mathbb{K}]\left[Z_{N}\right]$ are positive-definite symmetric $(N \times N)$ real matrices. Consequently, the reduced model of the finite element approximation is written as

$$
\mathbf{y}^{N}(t)=\left[Z_{N}\right] \mathbf{q}^{N}(t)
$$

in which the vector $\mathbf{q}^{N}(t) \in \mathbb{R}^{N}$ of the generalized coordinates verifies the nonlinear differential equation,

$$
\left[M_{N}\right] \ddot{\mathbf{q}}^{N}(t)+\left[D_{N}\right] \dot{\mathbf{q}}^{N}(t)+\left[K_{N}\right] \mathbf{q}^{N}(t)+\mathbf{F}_{\mathrm{NL}}^{N}\left(\mathbf{q}^{n}(t), \dot{\mathbf{q}}^{N}(t)\right)=\mathbf{F}^{N}(t)
$$

where, for all $\mathbf{q}$ and $\mathbf{p}$ in $\mathbb{R}^{N}$,

$$
\mathbf{F}_{\mathrm{NL}}^{N}(\mathbf{q}, \mathbf{p})=\left[Z_{N}\right]^{T} \mathbf{f}_{\mathrm{NL}}\left(\left[Z_{N}\right] \mathbf{q},\left[Z_{N}\right] \mathbf{p}\right)
$$

7. Observation of the discretized nonlinear elastodynamical system

The objective of this section is to define an observation of the mechanical system. From Section 2.3 , it can be deduced that Eq. (16) has a unique solution $t \mapsto \mathbf{y}(t)$ such that $\mathbf{y}$ and $\dot{\mathbf{y}}$ are square integrable vector-valued functions on $\mathbb{R}$. An approximation of this solution is computed using the reduced model of the finite element approximation defined by Eqs. (27) to (29). We introduce the energy $\widetilde{e}$ defined by

$$
\widetilde{e}=\int_{\mathbb{R}}\left(\frac{1}{2}\langle[\mathbb{M}] \dot{\mathbf{y}}(t), \dot{\mathbf{y}}(t)\rangle+\frac{1}{2}\langle[\mathbb{K}] \mathbf{y}(t), \mathbf{y}(t)\rangle\right) d t
$$


Let $\widehat{\mathbf{y}}(\omega)=\int_{\mathbb{R}} e^{-i \omega t} \mathbf{y}(t) d t$ be the Fourier Transform of $\mathbf{y}$. Using the Parseval formula, Eq. (30) yields

$$
\widetilde{e}=\int_{\mathbb{R}} h(\omega) d \omega=2 \int_{\mathbb{R}^{+}} h(\omega) d \omega
$$

in which $h(\omega)$ is the density of the energy associated with $\widetilde{e}$, related to the frequency band and which is written as

$$
h(\omega)=\frac{1}{2 \pi}\left\{\frac{1}{2}\left\langle\omega^{2}[\mathbb{M}] \widehat{\mathbf{y}}(\omega), \overline{\mathbf{y}}(\omega)\right\rangle+\frac{1}{2}\langle[\mathbb{K}] \widehat{\mathbf{y}}(\omega), \overline{\widehat{\mathbf{y}}(\omega)}\rangle\right\}
$$

where the overline denotes the complex conjugate.

Using the reduced model defined by Eqs. (27) to (29), the approximation $h^{N}(\omega)$ of $h(\omega)$ defined by Eq. (32) can be written as

$$
h^{N}(\omega)=\frac{1}{2 \pi}\left\{\frac{1}{2}\left\langle\omega^{2}\left[M_{N}\right] \widehat{\mathbf{q}}^{N}(\omega), \overline{\widehat{\mathbf{q}}^{N}(\omega)}\right\rangle+\frac{1}{2}\left\langle\left[K_{N}\right] \widehat{\mathbf{q}}^{N}(\omega), \overline{\widehat{\mathbf{q}}^{N}(\omega)}\right\rangle\right\}
$$

in which $\widehat{\mathbf{q}}^{N}(\omega)=\int_{\mathbb{R}} e^{-i \omega t} \mathbf{q}^{N}(t) d t$ is the Fourier Transform of $\mathbf{q}^{N}$.

8. Time discretization and numerical solvers

\subsection{Time-frequency numerical integration parameters and error functions}

In this section (1) we define the time-frequency numerical integration parameters for the numerical solvers related to the finite element model and to the reduced model, and (2) we introduce the error functions allowing the efficiency of the two reduced models constructed with the LIN-basis and with the POD-basis to be evaluated.

Let $B$ be the frequency band of analysis defined by

$$
B=\left[-\omega_{\max }, \omega_{\max }\right],
$$

in which $\omega_{\max }=2 \pi f_{\max }$ is the frequency such that

$$
\left|\int_{\mathbb{R}}\|\widehat{\mathbf{y}}(\omega)\|^{2} d \omega-\int_{-\omega_{\max }}^{\omega_{\max }}\|\widehat{\mathbf{y}}(\omega)\|^{2} d \omega\right| \leq \epsilon \quad,
$$

in which $\epsilon$ is an a priori given precision. Clearly, the band $B$ is such that $\underline{B}_{e} \cup B_{e} \subset B$. 
In order to define the error functions we introduce the frequency $\widetilde{\omega}_{\max }$ such that $\widetilde{\omega}_{\max } \leq \omega_{\max }$ and where $\left[0, \widetilde{\omega}_{\max }\right]$ is the frequency band of interest. We denote by $h_{\mathrm{LIN}}^{N}(\omega)$ the quantity $h^{N}(\omega)$ defined by Eq. (33) when the Hilbertian basis corresponds to the LIN-basis and by $h_{\mathrm{POD}}^{N}(\omega)$ when the POD-basis is used. We then defined the following error functions, depending on the dimension $N$ of the reduced model,

$$
\begin{gathered}
e_{\mathrm{REF}-\mathrm{LIN}}(N)=\frac{1}{\widetilde{\omega}_{\max }} \int_{0}^{\widetilde{\omega}_{\max }}\left(\log _{10} h(\omega)-\log _{10} h_{\mathrm{LIN}}^{N}(\omega)\right) d \omega, \\
e_{\mathrm{REF}-\mathrm{POD}}(N)=\frac{1}{\widetilde{\omega}_{\max }} \int_{0}^{\widetilde{\omega}_{\max }}\left(\log _{10} h(\omega)-\log _{10} h_{\mathrm{POD}}^{N}(\omega)\right) d \omega, \\
e_{\mathrm{LIN}-\mathrm{POD}}(N)=\frac{1}{\widetilde{\omega}_{\max }} \int_{0}^{\widetilde{\omega}_{\max }}\left(\log _{10} h_{\mathrm{LIN}}^{N}(\omega)-\log _{10} h_{\mathrm{POD}}^{N}(\omega)\right) d \omega,
\end{gathered}
$$

measuring, respectively, the error between the reference solution and the LIN-basis solution, the reference solution and the POD-basis solution and finally the LIN-basis solution and the POD-basis solution.

The integration time step is taken as $\Delta t=1 /\left(2 f_{\max }\right)$ and the time integration is $\widehat{T}=n_{\text {time }} \Delta t$ with $n_{\text {time }}$ a positive integer chosen as a power of 2 . The integration in $T=\mathbb{R}$ is approximated by an integration over the finite interval $\left[t_{0}, t_{1}\right]$ in which $t_{0}=-\widehat{T} / 2$ and $t_{1}=\widehat{T} / 2-\Delta t$. The sampling time points are $t_{k}=t_{0}+k \Delta t, k=0, \ldots, n_{\text {time }}-1$. To compute the Fourier Transform by FFT algorithm, the integration frequency step is taken as $\Delta \omega=2 \omega_{\text {max }} / n_{\text {freq }}$ with $n_{\text {freq }}=n_{\text {time }}$. The sampling frequency points are $\omega_{k}=$ $-\omega_{\max }+k \Delta \omega, k=0, \ldots, n_{\text {freq }}-1$. Equations (16) and (28) are integrated over $\left[t_{0}, t_{1}\right]$ using an implicit step by step time-integration method (Newmark scheme) with zero initial conditions at $t_{0}$. At each time point $t_{k}$ the nonlinear algebraic equation deduced from Eqs. (16) and (28) are solved using an iteration method (fixed point).

\subsection{Time integration parameters for the POD method}

Using Section 8.1 the numerical time integration of Eq. (16) yields $\mathbf{y}\left(t_{k}\right)$ for $k=0, \ldots, n_{\text {time }}-1$. The time discretization of Eq. (23) is then written as 


$$
[A] \simeq \Delta t \sum_{k=0}^{n_{\text {time }}-1}[H] \mathbf{y}\left(t_{k}\right)\left([H] \mathbf{y}\left(t_{k}\right)\right)^{T}
$$

\subsection{Numerical solver for the generalized eigenvalue problem related to the POD-basis}

The eigenvectors $\mathbf{z}_{1}, \ldots, \mathbf{z}_{\mathbf{N}}$ associated with the $N$ largest eigenvalues $\widehat{\lambda}_{1} \geq \ldots \geq \widehat{\lambda}_{N}$ of the generalized eigenvalue problem defined by Eq. (22) can nowadays be computed for very large generalized eigenvalue problems using an iterative algorithm based on the subspace iteration method or the Lanzcos method (see references $[17,18]$ ). Using such iteration methods, it can be seen that the amount of computational work is only due to the calculation of quantities such as $[H][S]$ and $[A][S]$ in which $[S]$ is rectangular $p \times \widetilde{N}$ matrix changing at each iteration of the algorithm. The number of iterations for convergence is generally small, for instance 10 or 20. The integer $\widetilde{N}$ is chosen in practice as $\widetilde{N}=\min \{2 N, N+8\}$, and consequently is small. The computation of $[H][S]$ has very low numerical cost because $[H]$ is a sparse matrix (stored as a sparse matrix and the computations being performed with this sparse structure). The computation of $[A][S]$ is also done with low cost using the following equation deduced from Eq. (39):

$$
[A][S] \simeq \Delta t \sum_{k=0}^{n_{\text {time }}-1}[H] \mathbf{y}\left(t_{k}\right)\left([H] \mathbf{y}\left(t_{k}\right)\right)^{T}[S]
$$

Note that the vectors $\mathbf{s}^{k}=[H] \mathbf{y}\left(t_{k}\right)$ are computed with a reasonable numerical cost and can generally be stored in core memory even for very large problems. The vectors $\left([H] \mathbf{y}\left(t_{k}\right)\right)^{T}[S]$ can be rewritten as $\mathbf{s}^{k^{T}}[S]$ and is a $1 \times \widetilde{N}$ matrix whose computation has a low numerical cost and which can be stored in core memory for very large problems. With such an algorithm the full square $p \times p$ matrix $[A]$ is never assembled.

\subsection{Numerical solver for the generalized eigenvalue problem related to the LIN-basis}

Note that the eigenvectors $\mathbf{z}_{1}, \ldots, \mathbf{z}_{\mathbf{N}}$ associated with the $N$ smallest eigenvalues $\widehat{\mu}_{1} \leq \ldots \leq \widehat{\mu}_{N}$ of the generalized eigenvalue problem defined by Eq. (20) are computed using the usual subroutines based on the iterative algorithms for sparse matrices. 
8.5. Numerical solver for the generalized eigenvalue problem using the snapshot method

In 1987, Sirovich published a paper [19] proposing the Snapshot method which is a numerical solver related to the generalized eigenvalue problem defined by Eq. (22). This method represented a great progress and it has been used extensively. The Snapshot method is efficient to reduce the computational work. Using the snapshot method any eigenvector $\mathbf{z}$ of the generalized eigenvalue problem defined by Eq. (22) is written as

$$
\mathbf{z}=\sum_{\alpha=1}^{\nu} b_{\alpha} \mathbf{y}\left(\theta_{\alpha}\right)
$$

in which $b_{\alpha}$ are the new unknowns and where $\left\{\theta_{1}, \ldots, \theta_{\nu}\right\}$ is a subset of the set $\left\{t_{0}, t_{1}, \ldots, t_{n_{\text {time }}-1}\right\}$, the vectors $\left\{\mathbf{y}\left(\theta_{1}\right), \ldots, \mathbf{y}\left(\theta_{\nu}\right)\right\}$ being a set of dependent or independent vectors in $\mathbb{R}^{p}$. Using the Eq. (41), the projection of Eq. (22) yields the following reduced generalized eigenvalue problem

$$
[\mathcal{A}] \mathbf{b}=\tilde{\lambda}[\mathcal{H}] \mathbf{b}
$$

in which $\mathbf{b}=\left(b_{1}, \ldots, b_{\nu}\right) \in \mathbb{R}^{\nu}$. If some of the vectors of the set $\left\{\mathbf{y}\left(\theta_{1}\right), \ldots, \mathbf{y}\left(\theta_{\nu}\right)\right\}$ are dependent, then the symmetric $\nu \times \nu$ matrices $[\mathcal{A}]$ and $[\mathcal{H}]$ are positive but not positive definite. Nevertheless, in this case, the reduced generalized eigenvalue problem can be solved with an adapted algorithm without difficulty. In addition, it can be shown that the components of these matrices are written as

$$
[\mathcal{A}]_{\beta \alpha}=\left\langle[A] \mathbf{y}\left(\theta_{\alpha}\right), \mathbf{y}\left(\theta_{\beta}\right)\right\rangle \quad, \quad[\mathcal{H}]_{\beta \alpha}=\left\langle[H] \mathbf{y}\left(\theta_{\alpha}\right), \mathbf{y}\left(\theta_{\beta}\right)\right\rangle
$$

Also,

$$
[\mathcal{A}]_{\beta \alpha}=\Delta t \sum_{k=0}^{n_{\text {time }}-1}\left\langle[H] \mathbf{y}\left(t_{k}\right), \mathbf{y}\left(\theta_{\beta}\right)\right\rangle\left\langle\left([H] \mathbf{y}\left(t_{k}\right)\right), \mathbf{y}\left(\theta_{\alpha}\right)\right\rangle
$$

Consequently the matrices $[\mathcal{A}]$ and $[\mathcal{H}]$ can be computed with low numerical cost for large systems, because the matrix $[H]$ is sparse as explained in Section 8.3. 
9. Numerical studies of the efficiency of the POD-basis with respect to the LIN-basis for reducing the model

This section deals with the application of the theory presented in the previous sections in order to compare the efficiency of the reduced model obtained with the LIN-basis and the POD-basis introduced in Sections 3.2 and 4 and for three types of nonlinearities. The continuous elastic system is constituted of two coupled subsystems. The first subsystem is a linear continuous elastic system constituted of a Timoshenko beam with added dissipation. For the second nonlinear subsystem three cases are considered. The first case is constituted of a nonsymmetric distributed nonlinearities, the second one of a symmetric distributed nonlinearities and the third one of four symmetrical linear elastic barriers (inducing shocks).

\subsection{Description of the first linear subsystem}

The geometrical properties of the beam are: length $1 \mathrm{~m}$, width $0.1 \mathrm{~m}$, height $0.1 \mathrm{~m}$. The boundary conditions are of a cantilever beam. The beam is homogeneous, isotropic, whose material properties are: density $7500 \mathrm{~kg} / \mathrm{m}^{3}$, Young' s modulus $2.1 \times 10^{10} \mathrm{~N} / \mathrm{m}^{2}$, Poisson's coefficient 0.3 , shearing correction factor $5 / 6$. The damping model is introduced by the model damping rate which is 0.02 for the first three modes of the uncoupled subsystem, 0.01 for the fourth mode and 0.005 for the others. The mean finite element model of the cantilever beam is constituted of 100 2-nodes Timoshenko beam elements. The first six computed eigenfrequencies of the uncoupled subsystem are 26.9, 162.7, 432.9, 794.1, 1219.2 and $1685.3 \mathrm{~Hz}$.

\subsection{Description of the nonlinearities for the second subsystem: nonlinearity 1}

The second subsystem is constituted of a distributed density of non-symmetric nonlinear stiffness producing forces transversally to the beam. At each finite element node of the mesh of the beam the function $\mathbf{f}_{\mathrm{NL}}$ defined in Eq. (16) is then independent of the velocity and is constructed using

$$
f_{\mathrm{NL}}(y)= \begin{cases}0 & , y \leq 0 \\ k_{0} y^{3} & , y>0\end{cases}
$$


with $k_{0}=2.9301 \times 10^{18} \mathrm{~N} / \mathrm{m}$.

\subsection{Description of the nonlinearities for the second subsystem: nonlinearity 2}

The second subsystem is constituted of a distributed density of symmetric nonlinear stiffness producing forces transversally to the beam. At each finite element node of the mesh of the beam the function $\mathbf{f}_{\mathrm{NL}}$ defined in Eq. (16) is then independent of the velocity and is constructed using

$$
f_{\mathrm{NL}}(y)=k_{1} y^{3} \quad, \quad \forall y \in \mathbb{R}
$$

with $k_{1}=2.9301 \times 10^{18} \mathrm{~N} / \mathrm{m}$.

\subsection{Description of the nonlinearities for the second subsystem: nonlinearity 3}

The second subsystem can be viewed as boundary conditions applied to the cantilever beam at four points in which the motion is limited by four elastic barriers distant of $\varepsilon$, in both sides of the beam. The gap $\varepsilon$ is written as $\varepsilon=a \eta$ in which $\eta=10^{-6}$ and where the amplitude $a$ of the vector load $\mathbf{f}(t)$ defined by Eq. (17) is equal to 1 . The four elastic barriers are placed at the following distance from the fixed end: $0.14,0.37,0.60$ and 0.82 . The elasticity constant of the barrier is $\underline{k}_{b}=10^{7} \mathrm{~N} / \mathrm{m}$. The function $\mathbf{f}_{\mathrm{NL}}$ defined in Eq. (16) is then independent of the velocity and, for each elastic barrier, is constructed using

$$
f_{\mathrm{NL}}(y)=\left\{\begin{array}{ll}
0, & |y| \leq \varepsilon \\
k_{b}(y-\varepsilon \operatorname{sign}(y)), & |y|\rangle \varepsilon
\end{array},\right.
$$

in which $y$ is the transversal displacement at a given elastic barrier.

\subsection{Description of the excitation force}

The vector load is defined by Eq. (17). The amplitude $a$ is equal to 1 . The force is a point force applied at the free end of the beam. The impulse function $g$ is such that

$$
g(t)=\frac{1}{\pi t}\left\{\sin \left(t\left(\Omega_{c}+\Delta \Omega / 2\right)\right)-\sin \left(t\left(\Omega_{c}-\Delta \Omega / 2\right)\right)\right\},
$$


whose Fourier Transform is $\widehat{g}(\omega)=\mathbf{1}_{\underline{B}_{e} \cup B_{e}}$ in which the frequency band $B_{e}$ is defined by Eq. (18). We introduce the bandwidth $\Delta f$ in Hertz and the central frequency $f_{c}$ in Hertz such that $\Delta \Omega=2 \pi \Delta f$ and $\Omega_{c}=2 \pi f_{c}$ Two cases are considered for the excitation:

Narrow band excitation $\Delta f=200 \mathrm{~Hz}$ and $f_{c}=1180 \mathrm{~Hz}$. Consequently, the frequency band of excitation is close to the fifth eigenfrequency of the first linear subsystem.

Broad band excitation $\Delta f=1400 \mathrm{~Hz}$ and $f_{c}=701 \mathrm{~Hz}$. Consequently, the frequency band of excitation contains the first five eigenfrequencies of the first linear subsystem.

\subsection{Numerical integration parameters}

This section deals with the numerical integration parameters defined in Section 8.1. For all the numerical results presented below, the value of $f_{\max }$ has been calculated in order to obtain a good accuracy for the time integration scheme in the three types of nonlinearities considered. The value necessary to reach this accuracy is $12000 \mathrm{~Hz}$.

On the other hand a convergence analysis has been performed with respect to the time duration $\widehat{T}$ in order that the coupled system be at rest for $t=\widehat{T} / 2$ with a good accuracy. In this case, Eq. (35) is verified with a good accuracy. This duration is defined by the value of $n_{\text {time }}$ whose necessary value is 32768 .

\subsection{Efficiency of the LIN-basis and POD-basis compared with the reference solution}

The reference solution is defined as the solution of Eq. (16). The solution corresponding to the reduced model defined by Eqs. (27), (28), (29) and (33) and constructed using the LIN-basis (see Section 5.2) will be called LIN-solution. The solution corresponding to the reduced model defined by Eqs. (27), (28), (29) and (33) and constructed using the POD-basis (see Section 5.3) will be called POD-solution.

In this section we compare the convergence of the LIN-solution and the POD-solution in function of the dimension $N$ of the reduction with respect to the reference solution using the error functions defined by Eqs. (36) and (37). The comparison between the LIN-solution and the POD-solution in function of $N$ is given by the error function defined by Eq. (38). 
Below we present six cases (three different types of nonlinearities and two cases of excitations) that are analyzed to show the comparison of the two bases.

9.7.1. Nonlinearity 1 with broad band excitation $[0,1400] \mathrm{Hz} . \quad$ Figure 1 displays the graph of the function $f \mapsto \log _{10} h(2 \pi f)$ defined by Eq. (32) over [0, 8000] Hz for the reference solution.

Figure 2 displays the graph of the function $j \mapsto \log _{10}\left(\widehat{\lambda}_{j} / \widehat{\lambda}_{1}\right)$ in which $\widehat{\lambda}_{j}$ are the eigenvalues defined in Section 5.3. This figure shows the decreasing speed of the eigenvalues which is related to the rate of convergence of the POD-solution with respect to $N$.

For illustrating the convergence in the frequency domain we show the results for a given $N$. Figure 3 corresponds to $N=5$ and shows three curves related to the reference solution (thick solid line), LIN-solution (red thin solid line) and POD-solution (blue thin solid line) . It should be noted that the thin solid lines may be superposed and hence some of them are not visible.

The error functions are computed for $\widetilde{\omega}_{\max }=2 \pi \times 8000 \mathrm{rad} / \mathrm{s}$. Figure 4 displays the three error functions, dimensionless, allowing the efficiency of the LIN-solution and the POD-solution in function of dimension $N$ of the reduced model to be carried out. The analysis of this figure shows that the LIN-basis and the POD-basis have the same efficiency with respect to the dimension $N$ of the reduction in the frequency band $[0,8000] \mathrm{Hz}$, the convergence being reached for $N=20$. We mean by the same efficiency the fact that the two bases need the same value of $N$ to get convergence.

9.7.2. Nonlinearity 1 with narrow band excitation $[1080,1280] \mathrm{Hz}$. Figure 5 displays the graph of the function $f \mapsto \log _{10} h(2 \pi f)$ over $[0,5000] \mathrm{Hz}$ for the reference solution. Figure 6 displays the graph of $j \mapsto \log _{10}\left(\widehat{\lambda}_{j} / \widehat{\lambda}_{1}\right)$. Figure 7 corresponds to $N=5$. Henceforth, the convention for the lines is always the same.

The error functions are computed for $\widetilde{\omega}_{\max }=2 \pi \times 5000 \mathrm{rad} / \mathrm{s}$. Figure 8 displays the three error functions allowing the efficiency of the LIN-solution and the POD-solution in function of dimension $N$ of the reduced model to be carried out. The analysis of this figure shows that the LIN-basis and the POD-basis have the same efficiency with respect to $N$ in the frequency band $[0,5000] \mathrm{Hz}$. Nevertheless the POD-basis is more efficient than the LIN-basis for $N \leq 10$, 
but for this values of $N$ solution has not yet converged, that is achieved for $N=15$.

9.7.3. Nonlinearity 2 with broad band excitation $[0,1400] \mathrm{Hz}$. Figure 9 corresponds to $N=5$. The convention for the lines is always the same.

The error functions are computed for $\widetilde{\omega}_{\max }=2 \pi \times 8000 \mathrm{rad} / \mathrm{s}$. Figure 10 displays the three error functions allowing the efficiency of the LIN-solution and the POD-solution in function of dimension $N$ of the reduced model to be carried out. The analysis of this figure shows that the LIN-basis and the POD- basis have the same efficiency with respect to $N$ in the frequency band $[0,8000] \mathrm{Hz}$. The convergence is reached for $N=20$.

9.7.4. Nonlinearity 2 with narrow band excitation [1080,1280] Hz. Figures 11 correspond to $N=5$. The convention for the lines is always the same.

The error functions are computed for $\widetilde{\omega}_{\max }=2 \pi \times 5000 \mathrm{rad} / \mathrm{s}$. Figure 12 displays the three error functions allowing the efficiency of the LIN-solution and the POD-solution in function of dimension $N$ of the reduced model to be carried out. The analysis of this figure shows that the LIN-basis and the POD- basis have the same efficiency with respect to $N$ in the frequency band $[0,5000] \mathrm{Hz}$. The convergence is reached for $N=20$.

9.7.5. Nonlinearity 3 with broad band excitation $[0,1400] \mathrm{Hz}$. Figures 13 correspond to $N=5$. The convention for the lines is always the same.

The error functions are computed for $\widetilde{\omega}_{\max }=2 \pi \times 8000 \mathrm{rad} / \mathrm{s}$. Figure 14 displays the three error functions allowing the efficiency of the LIN-solution and the POD-solution in function of dimension $N$ of the reduced model to be carried out. The analysis of this figure shows that the LIN-basis and the POD- basis have the same efficiency with respect to $N$ in the frequency band $[0,8000] \mathrm{Hz}$. Nevertheless it can be seen that the LIN-basis is more efficient than the POD-basis for $N \leq 14$ corresponding to values of $N$ for which solutions has not yet converged. Convergence is reached for $N=30$. 
9.7.6. Nonlinearity 3 with narrow band excitation $[1080,1280] \mathrm{Hz}$. Figure 15 corresponds to $N=5$. The convention for the lines is always the same.

The error functions are computed for $\widetilde{\omega}_{\max }=2 \pi \times 5000 \mathrm{rad} / \mathrm{s}$. Figure 16 displays the three error functions allowing the efficiency of the LIN-solution and the POD-solution in function of dimension $N$ of the reduced model to be carried out. The analysis of this figure shows that the LIN-basis and the POD- basis have the same efficiency with respect to $N$ in the frequency band $[0,5000] \mathrm{Hz}$. Nevertheless it can be seen that the POD-basis is more efficient than the LIN-basis for $N \leq 15$ corresponding to values of $N$ for which solutions has not yet converged. Convergence is reached for $N=20$.

\section{Conclusions}

This paper has been devoted to the analysis of the efficiency of the reduced models constructed using the POD-basis and the LIN-basis in nonlinear dynamics for continuous elastic systems. The efficiency of the POD-basis or the LIN-basis is related to the rate of convergence in the frequency domain of the solution constructed with the reduced model with respect to its dimension. A basis will be more efficient than another if the reduced-order solution of the Galerkin projection converges to the solution of the dynamical system more rapidly than the reduced-order solution of the other. It can be concluded that the POD-basis is not more efficient than the LIN-basis for the six examples treated.

\section{REFERENCES}

1. Loève, M. Probability Theory (Third edition). Van Nostrand: New York, 1963.

2. Holmes P, Lumley JL, Berkooz G. Turbulence, Coherent Structures, Dynamical Systems and Symmetry. Cambridge University Press: Cambridge, 1997.

3. Kunisch, E, Volkwein, S. Galerkin proper orthogonal decomposition methods for parabolic problems. Numerische Mathematik 2001; 90(1): 117-148.

4. Kunisch, E, Volkwein, S. Galerkin proper orthogonal decomposition methods for a general equation in fluid dynamics. SIAM Journal of Numerical Analysis 2002; 40(1): 233-253.

5. Azeez MFA, Vakakis AF. Proper orthogonal decomposition (POD) of a class of vibroimpact oscillations. Journal of Sound and Vibration 2001; 240(5):859-889.

6. Meyer M, Matthies HG. Efficient model reduction in non-linear dynamics using the Karhunen-Loéve expansion and dual-weighted-residual methods. Computational Mechanics 2003; 31:179-191.

7. Park HM, Cho DH. The use of the Karhunen-Loève decomposition for the modeling of distributed parameters systems. Chemical Engineering Science 1996; 51(1):81-98. 
8. Rowley CW, Marsden JE. Reconstruction equations and the Karhunen-Loève expansion for systems with symmetry. Physica D 2000;142:1-19.

9. Wolter C, Trindade MA, Sampaio R. Reduced-order model for an impacting beam using the KarhunenLoève expansion. Tendências em Matemática Aplicada e Computacional (TEMA) 2002; 3(2): 217-226.

10. Trindade MA, Wolter C, Sampaio R. Karhunen-Loève decomposition of coupled axial/bending vibrations of beams subject to impacts. Journal of Sound and Vibration 2005; 279:1015-1036.

11. Dautray R, Lions JL. Mathematical Analysis and Numerical Methods for Science and Technology. Vol 1-2-3. Springer-Verlag: Berlin, 1992.

12. Ohayon R, Soize C. Structural Acoustics and Vibration: Mechanical Models, Variational Formulations and Discretization. Academic Press: San Diego, 1998.

13. Bellizzi S, Sampaio R. POMs analysis of randomly vibrating systems obtained from Karhunen-Loève expansion. Journal of Sound and Vibration 2006; 297:774-793.

14. Soize C. Reduced models in the medium frequency range for general dissipative structural-dynamics systems. European Journal of Mechanics A/Solids 1998; 17(4):657-685.

15. Guelfand IM, Vilenkin NY. Les Distributions. Tome 4. Application de l'Analyse Harmonique. Dunod: Paris, 1967.

16. Zienkiewicz OC, Taylor RL. Finite Element Method. Vol 1-2-3. Butterworth-Heinomann:London, 2000.

17. Chatelin F. Eigenvalues of Matrices. Wiley: New York, 1993.

18. Bathe KJ, Wilson EL. Numerical Methods in Finite Element Analysis. Prentice Hall: New York, 1976.

19. Sirovich L. Turbulence and the dynamics of coherent structures, Part I: coherent structures. Quartely of Applied Mathematics 1987; 45(3):561-571.

\section{Acknowledgments}

This work was founded by the Brazilian Agency Conselho Nacional de Desenvolvimento

Científico e Tecnológico (CNPq), by the International Cooperation Project Capes-Cofecub, $n^{\circ} 476 / 04$, and Faperj. 


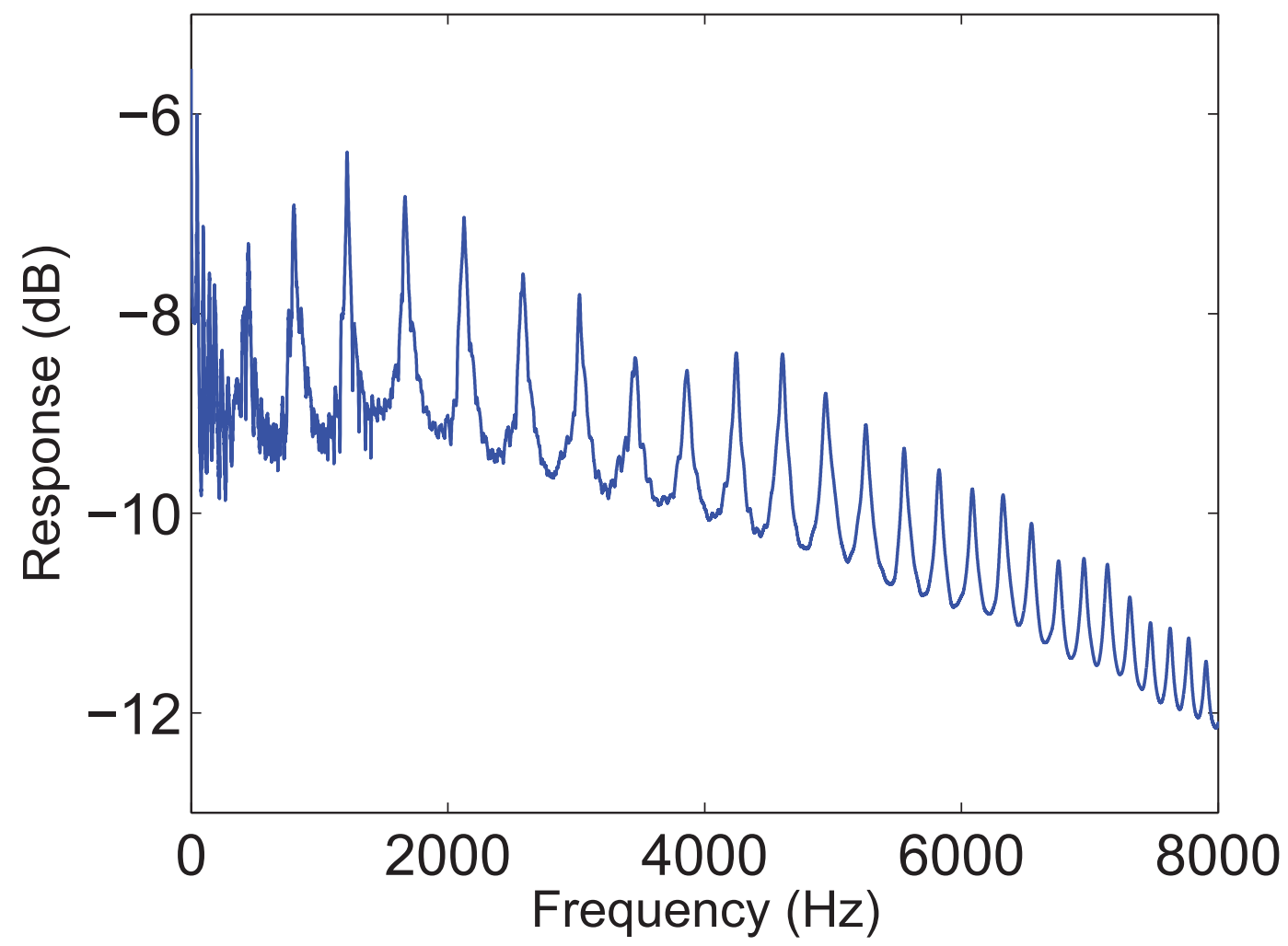

Figure 1: Nonlinearity 1 - Broad band [0,1400] Hz: Graph of $f \mapsto \log _{10} h(2 \pi f)$ for the reference solution. Horizontal axis frequency in Hertz. Vertical axis $f \mapsto$ $\log _{10} h(2 \pi f)$. 


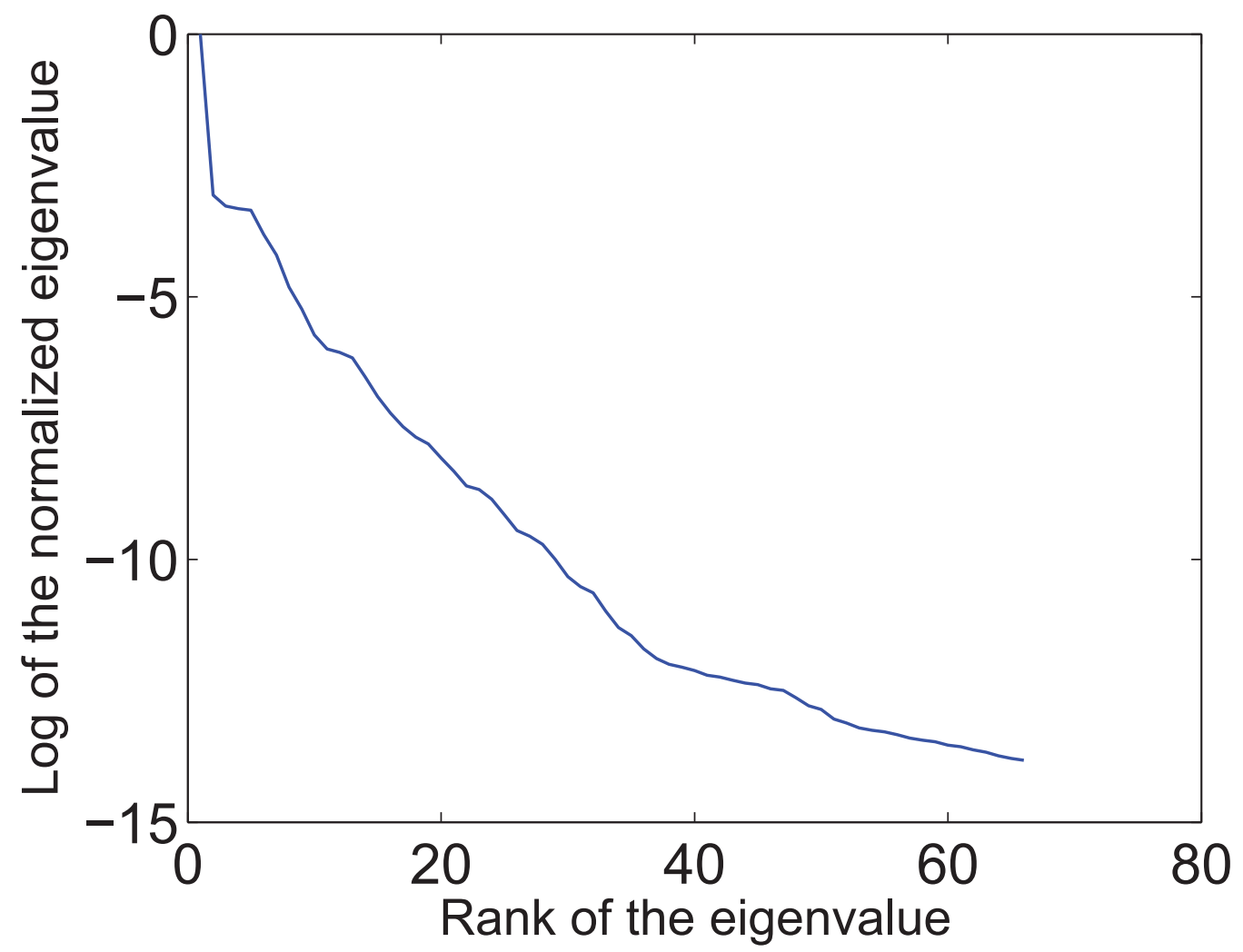

Figure 2: Nonlinearity 1 - Broad band $[0,1400] \mathrm{Hz}$ : Graph of $j \mapsto \log _{10}\left(\widehat{\lambda}_{j} / \widehat{\lambda}_{1}\right)$. Horizontal axis rank $j$ of the eigenvalue. Vertical axis $\log _{10}\left(\widehat{\lambda}_{j} / \widehat{\lambda}_{1}\right)$. 


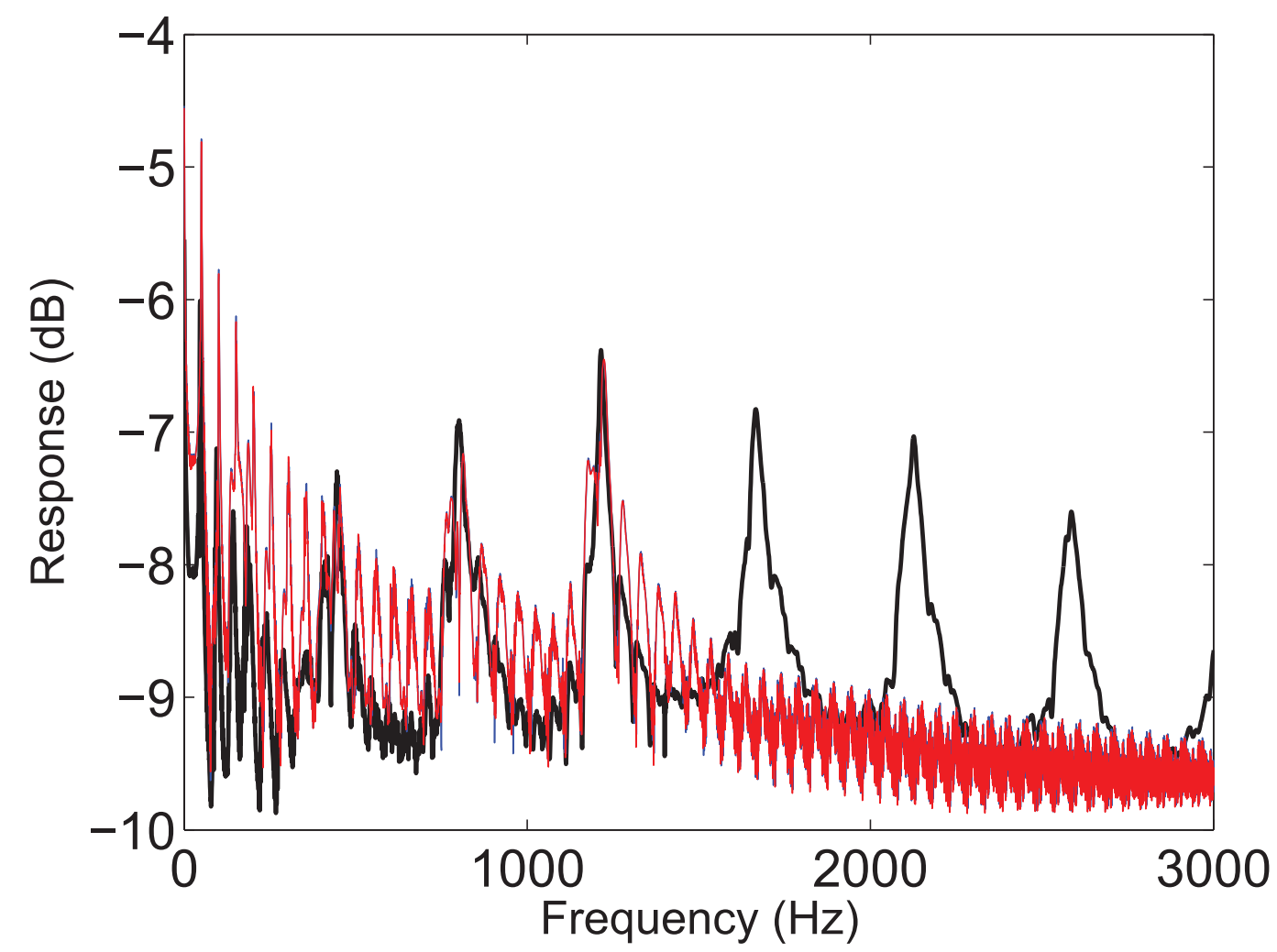

Figure 3: Nonlinearity 1 - Broad band $[0,1400] \mathrm{Hz}-N=5$ : Graph of $f \mapsto$ $\log _{10} h(2 \pi f)$ for the reference solution (black line), RML-solution (red line) and RMNL-solution (blue line). Horizontal axis frequency in Hertz. Vertical axis $f \mapsto \log _{10} h(2 \pi f)$. 


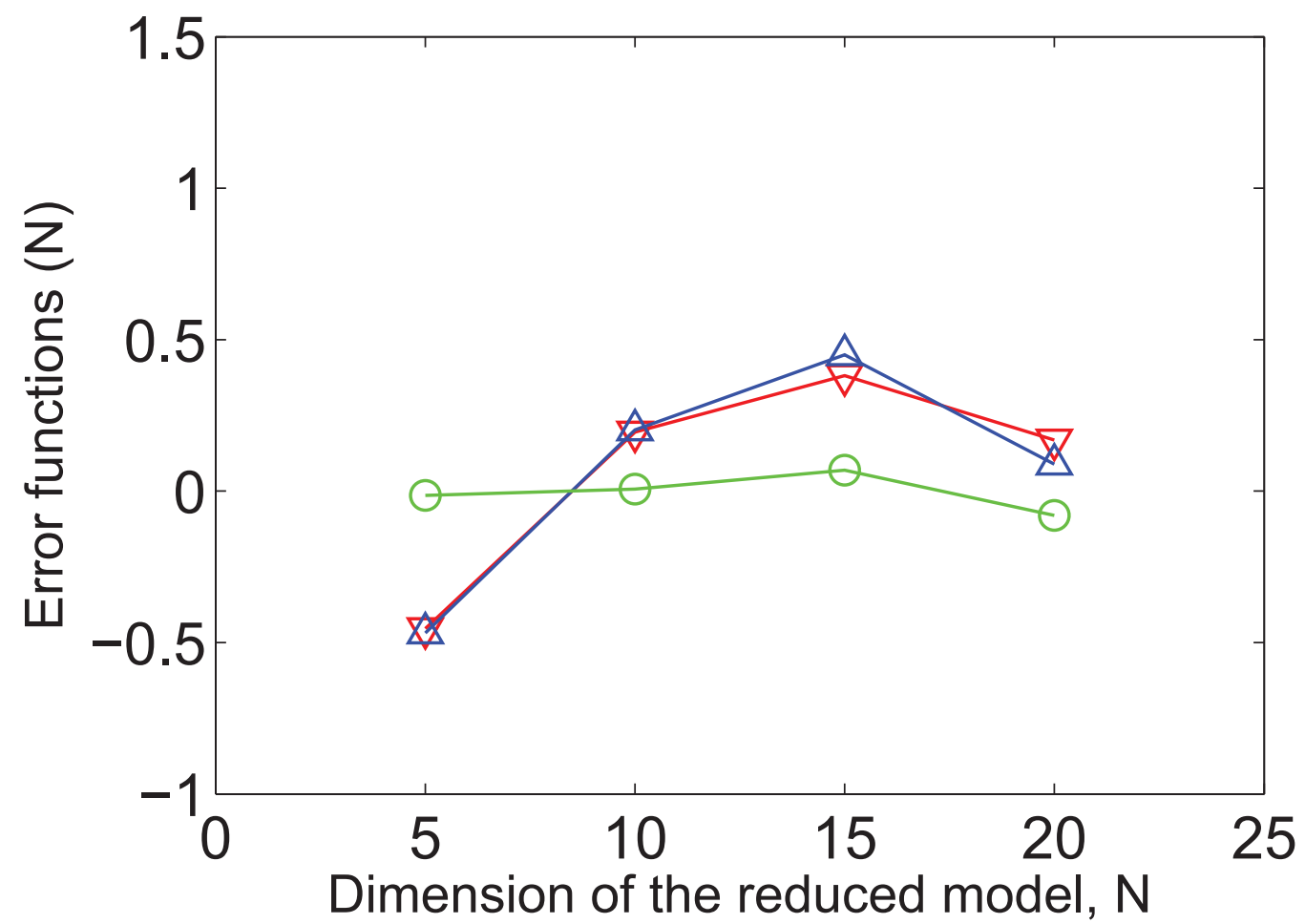

Figure 4: Nonlinearity 1 - Broad band [0, 1400] Hz - Efficiency of the LIN-solution and POD-solution in function of dimension $N$ of the reduced model. Graphs of the error functions versus $N$ : $e_{\text {REF-LIN }}(N)$ (triangle down), $e_{\text {REF-POD }}(N)$ (triangle up), $e_{\mathrm{LIN}-\mathrm{POD}}(N)$ (circle). 


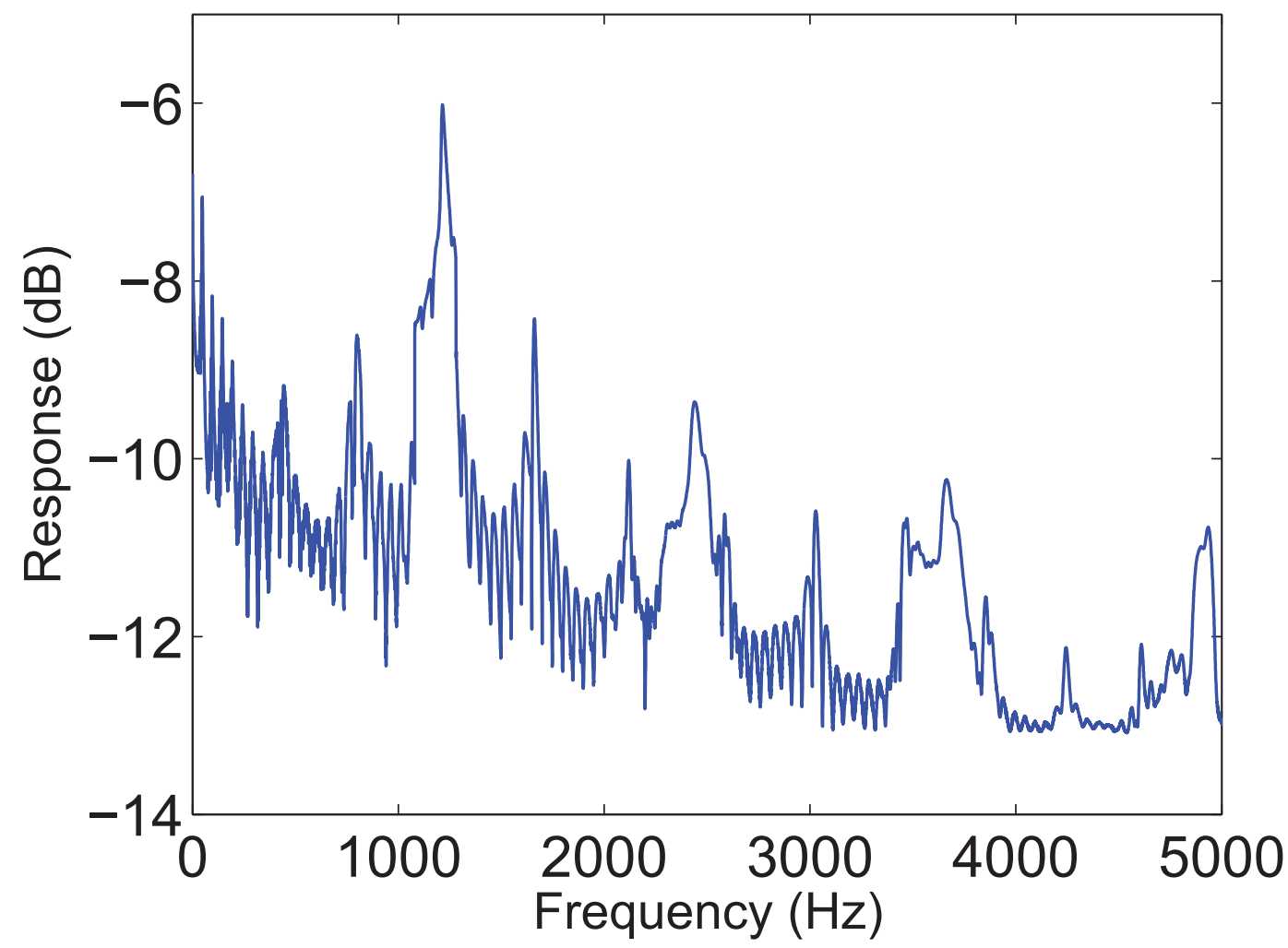

Figure 5: Nonlinearity 1 - Narrow band $[1080,1280]$ Hz: Graph of $f \mapsto \log _{10} h(2 \pi f)$ for the reference solution. Horizontal axis frequency in Hertz. Vertical axis $f \mapsto$ $\log _{10} h(2 \pi f)$. 


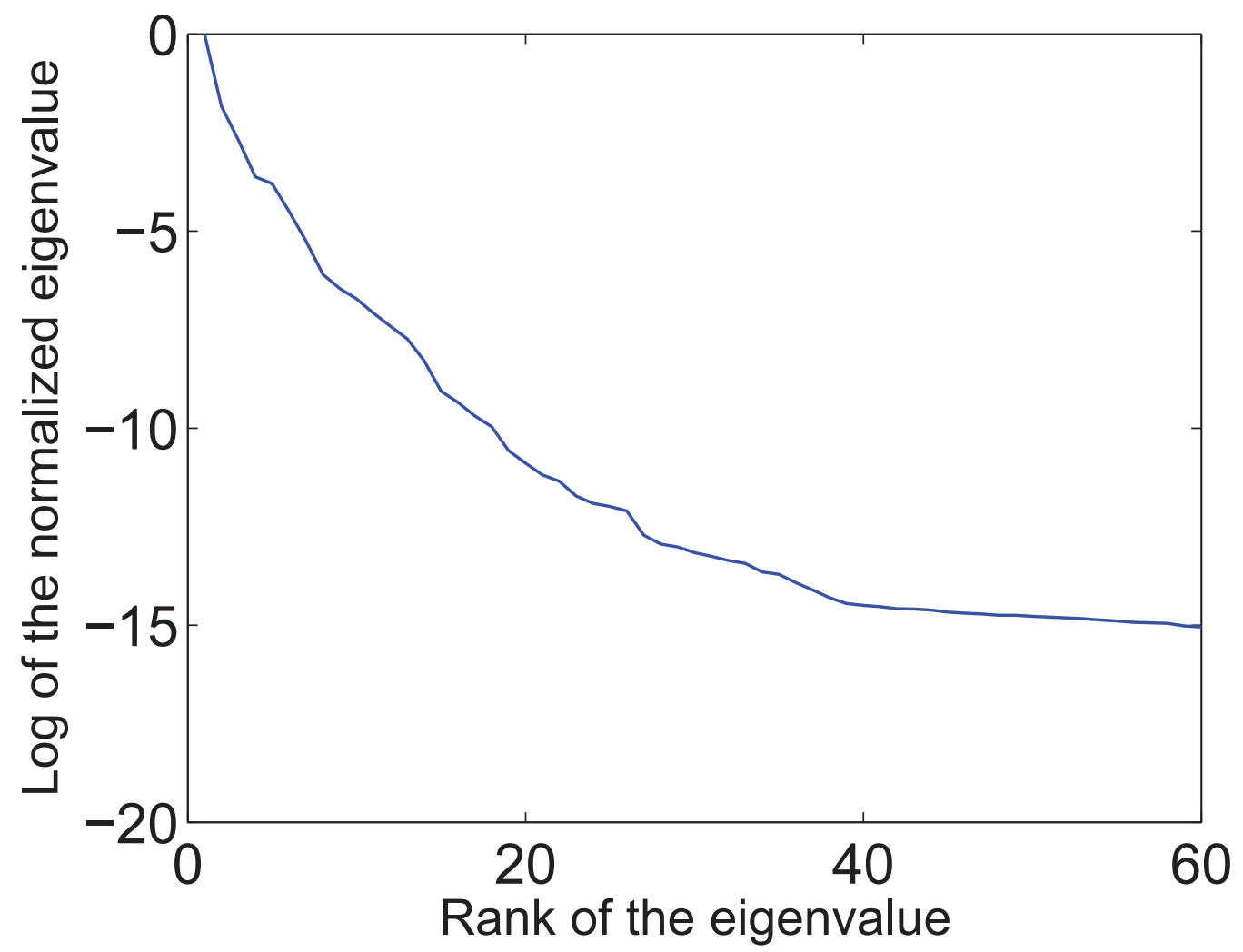

Figure 6: Nonlinearity 1 - Narrow band [1080,1280] Hz: Graph of $j \mapsto$ $\log _{10}\left(\widehat{\lambda}_{j} / \widehat{\lambda}_{1}\right)$. Horizontal axis rank $j$ of the eigenvalue. Vertical axis $\log _{10}\left(\widehat{\lambda}_{j} / \widehat{\lambda}_{1}\right)$. 


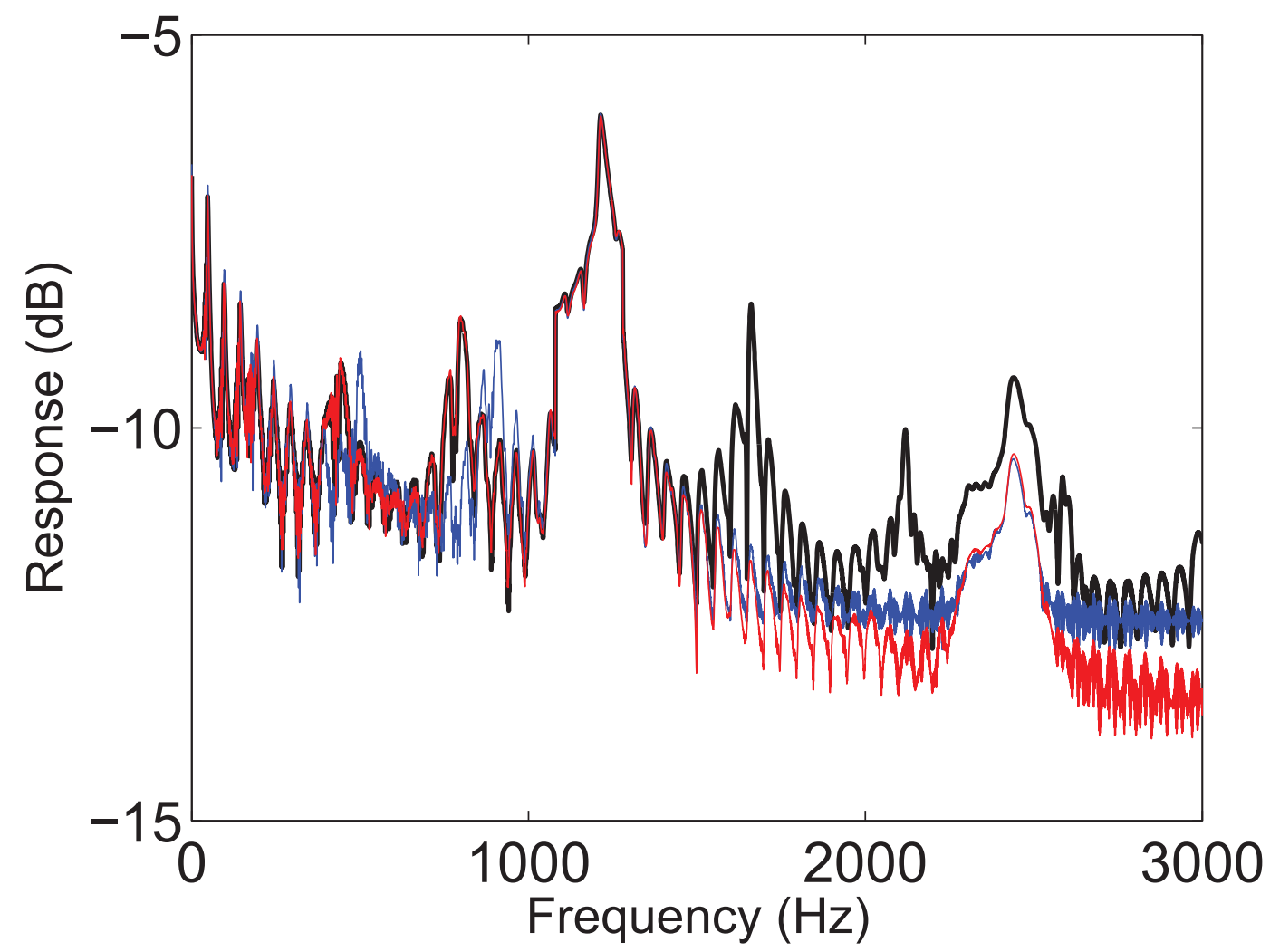

Figure 7: Nonlinearity 1 - Narrow band $[1080,1280] \mathrm{Hz}-N=5$ : Graph of $f \mapsto$ $\log _{10} h(2 \pi f)$ for the reference solution (black line), RML-solution (red line) and RMNL-solution (blue line). Horizontal axis frequency in Hertz. Vertical axis $f \mapsto \log _{10} h(2 \pi f)$. 


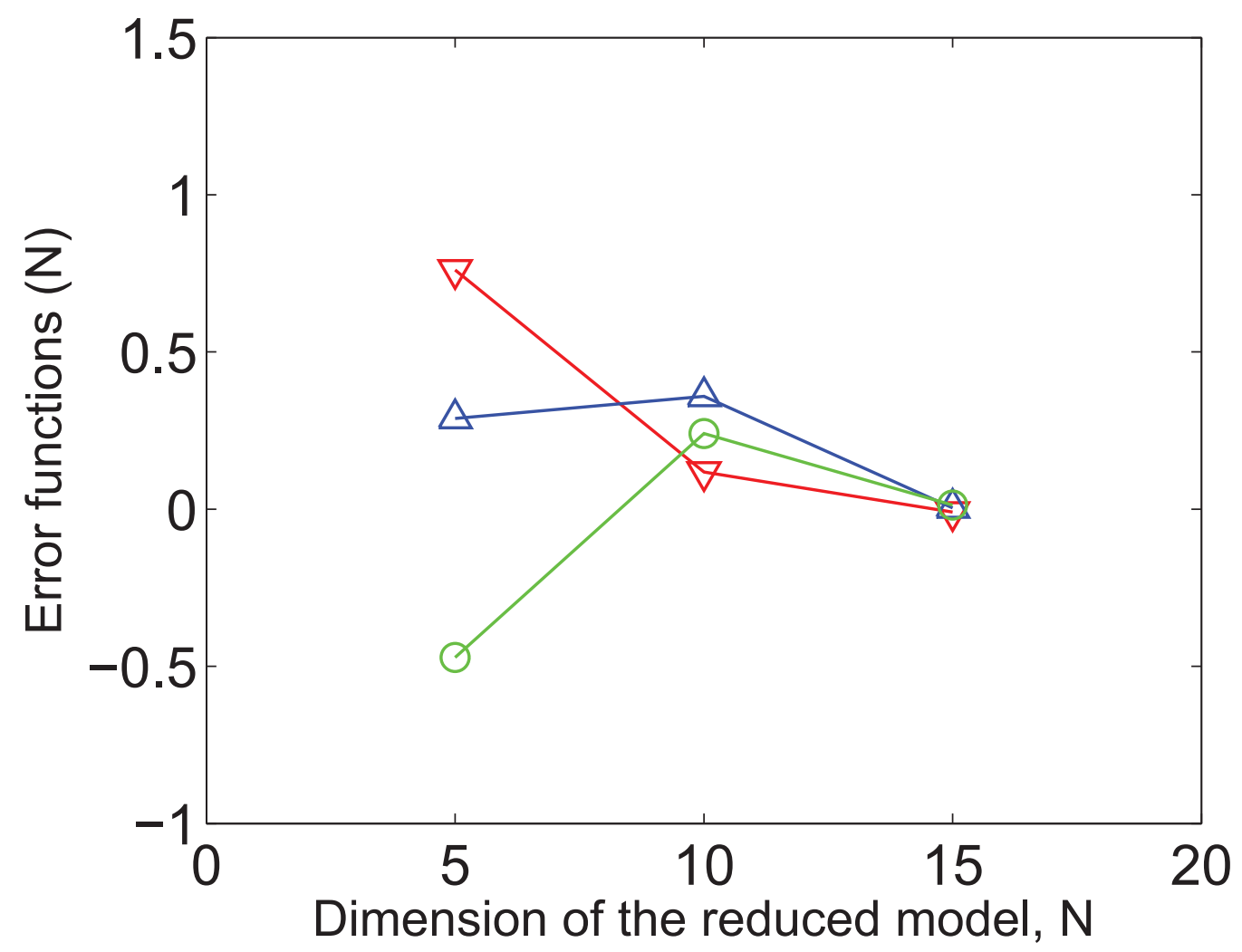

Figure 8: Nonlinearity 1 - Narrow band [1080, 1280] Hz - Efficiency of the LINsolution and POD-solution in function of dimension $N$ of the reduced model. Graphs of the error functions versus $N: e_{\text {REF-LIN }}(N)$ (triangle down), $e_{\text {REF-POD }}(N)$ (triangle up), $e_{\text {LIN-POD }}(N)$ (circle). 


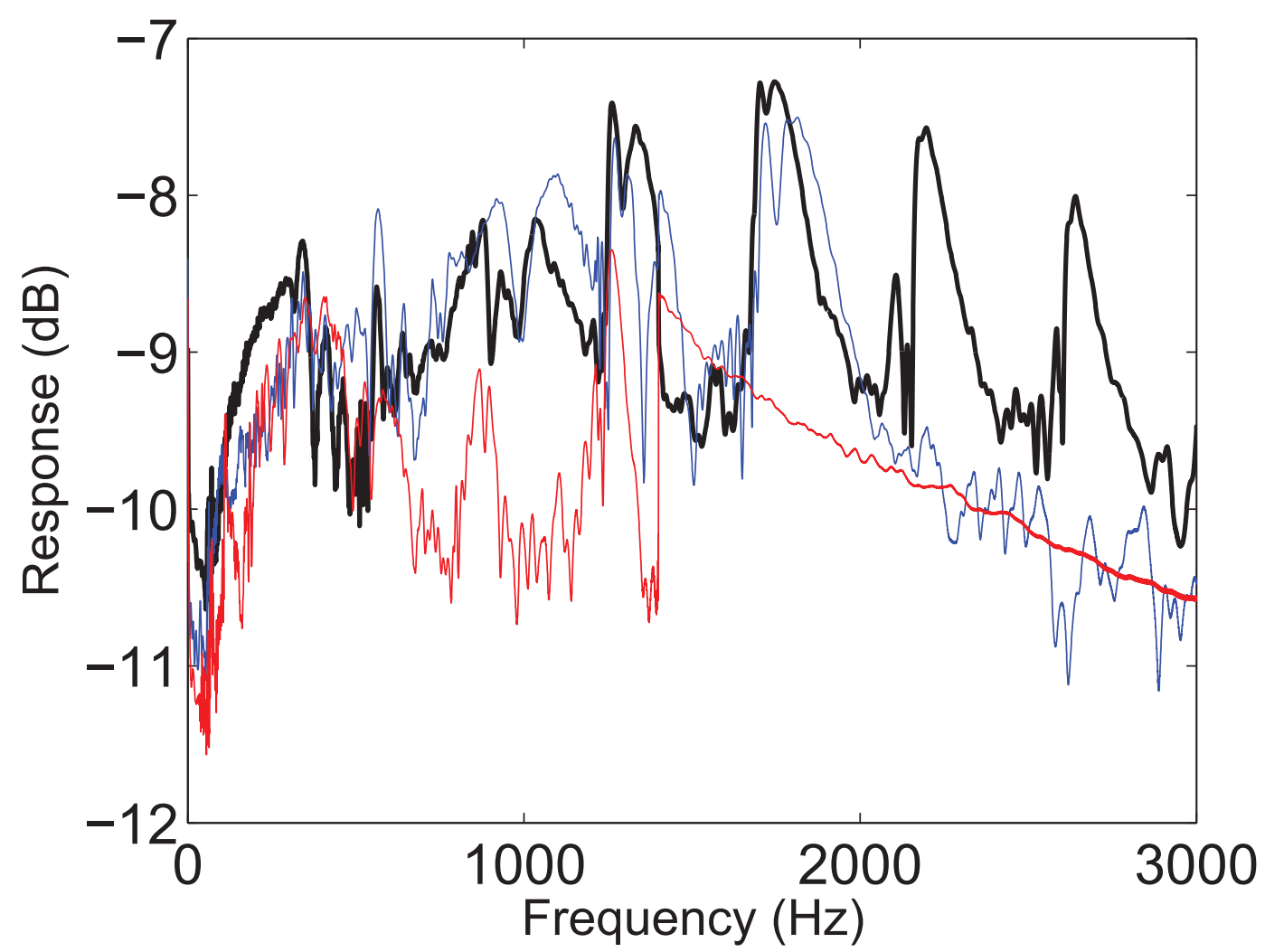

Figure 9: Nonlinearity 2 - Broad band $[0,1400] \mathrm{Hz}-N=5$ : Graph of $f \mapsto$ $\log _{10} h(2 \pi f)$ for the reference solution (black line), RML-solution (red line) and RMNL-solution (blue line). Horizontal axis frequency in Hertz. Vertical axis $f \mapsto \log _{10} h(2 \pi f)$. 


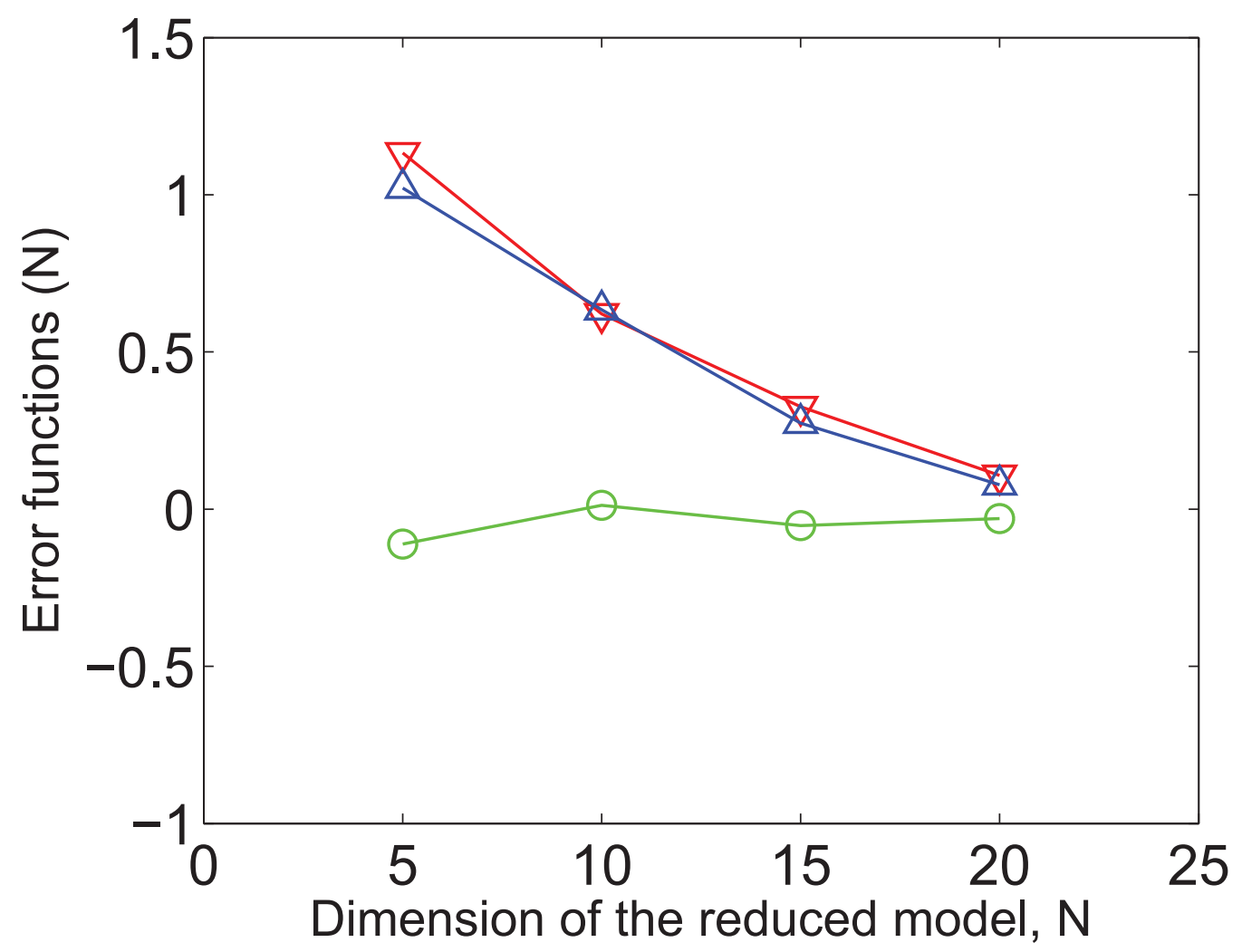

Figure 10: Nonlinearity 2 - Broad band [0, 1400] Hz - Efficiency of the LIN-solution and POD-solution in function of dimension $N$ of the reduced model. Graphs of the error functions versus $N$ : $e_{\text {REF-LIN }}(N)$ (triangle down), $e_{\text {REF-POD }}(N)$ (triangle up), $e_{\mathrm{LIN}-\mathrm{POD}}(N)$ (circle). 


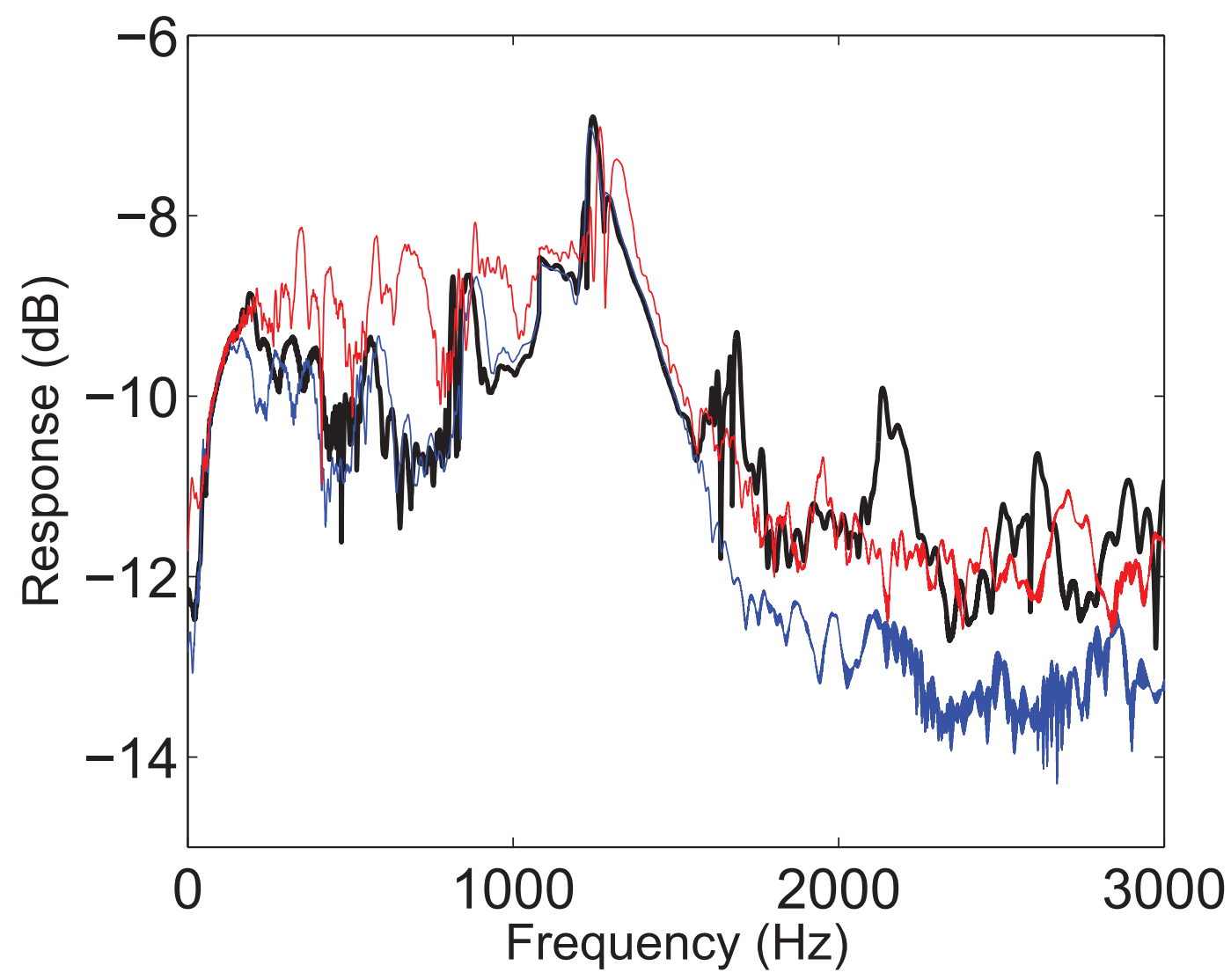

Figure 11: Nonlinearity 2 - Narrow band [1080,1280] $\mathrm{Hz}-N=5$ : Graph of $f \mapsto \log _{10} h(2 \pi f)$ for the reference solution (black line), RML-solution (red line) and RMNL-solution (blue line). Horizontal axis frequency in Hertz. Vertical axis $f \mapsto \log _{10} h(2 \pi f)$. 


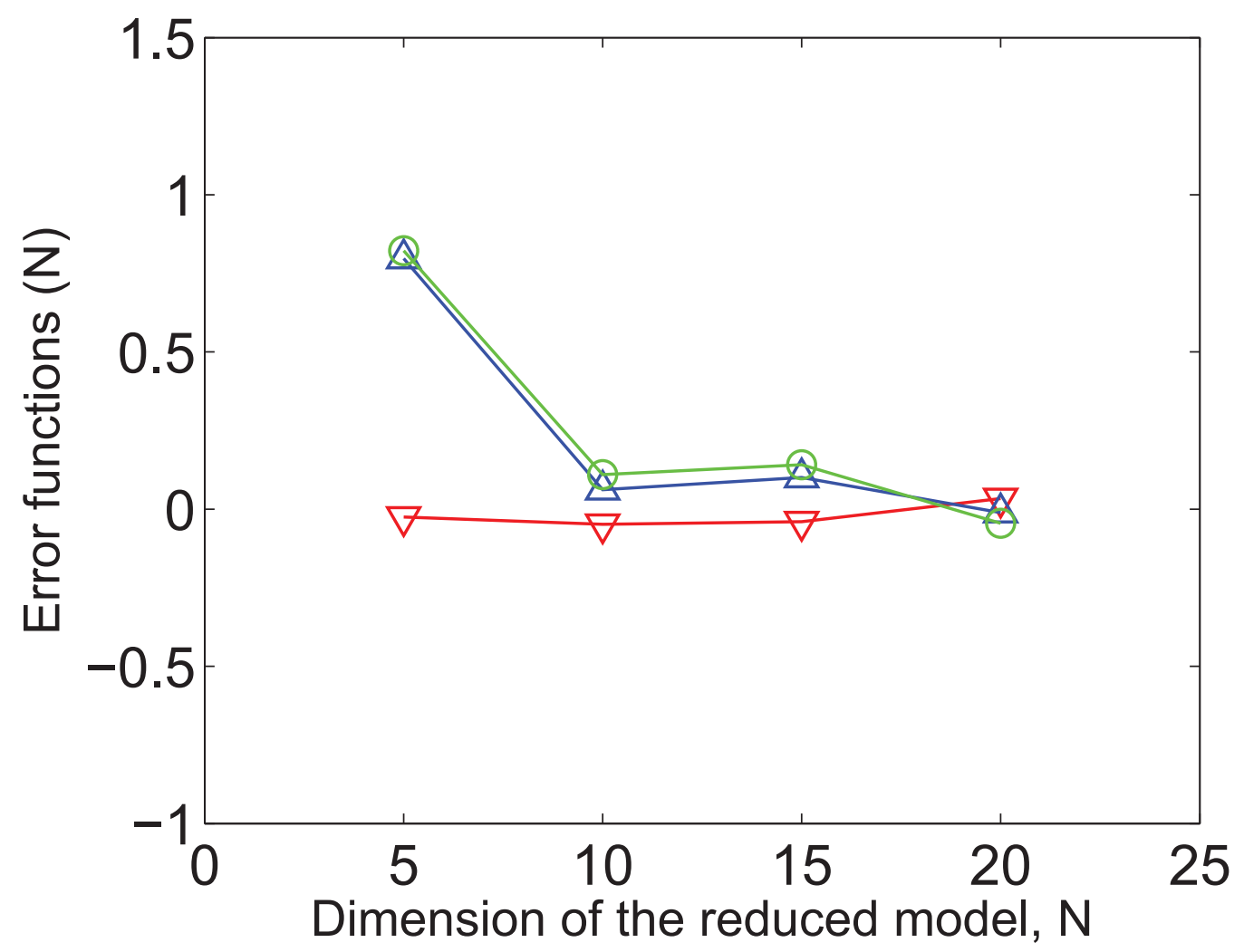

Figure 12: Nonlinearity 2 - Narrow band [1080, 1280] Hz - Efficiency of the LINsolution and POD-solution in function of dimension $N$ of the reduced model. Graphs of the error functions versus $N: e_{\text {REF-LIN }}(N)$ (triangle down), $e_{\text {REF-POD }}(N)$ (triangle up), $e_{\text {LIN-POD }}(N)$ (circle). 


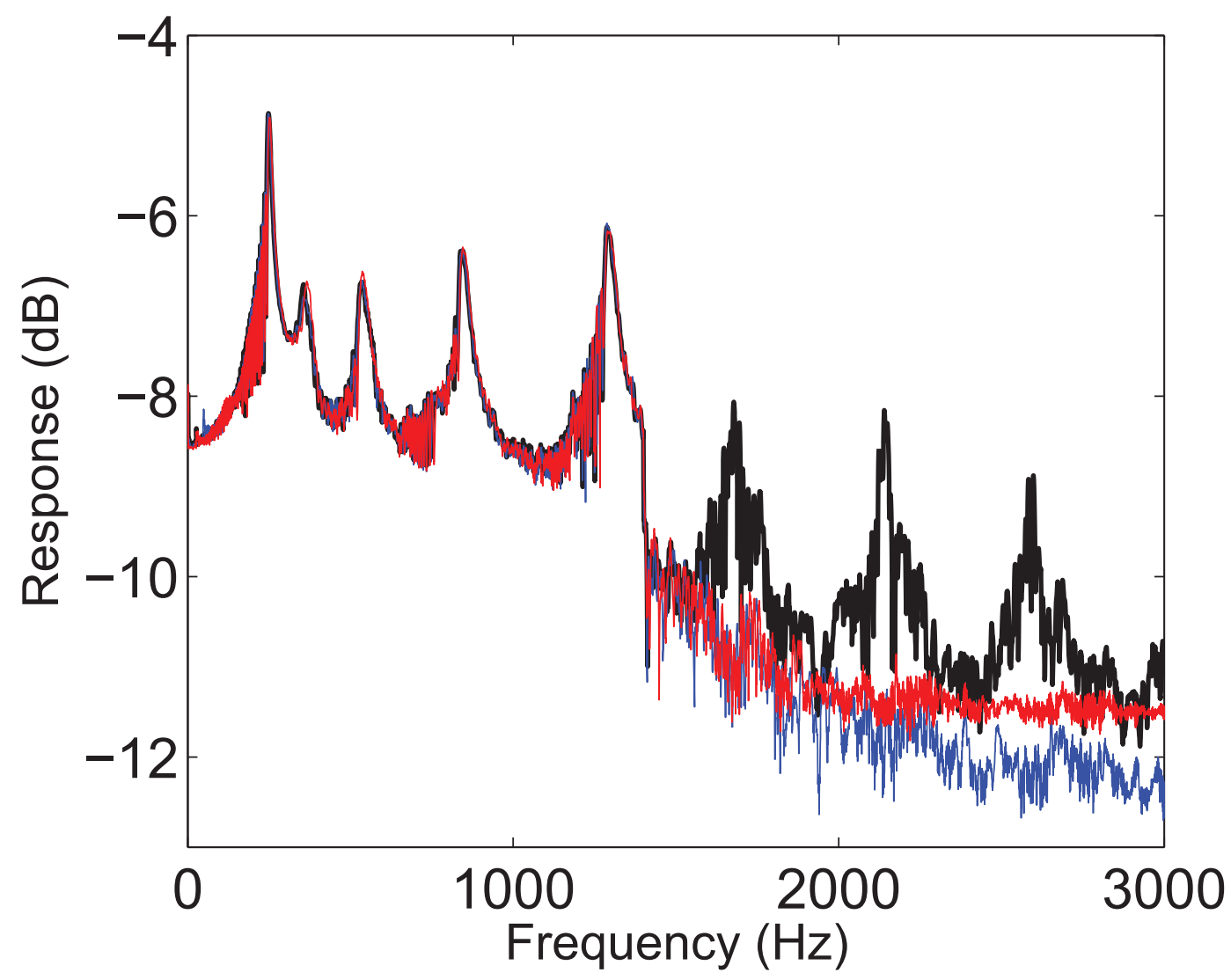

Figure 13: Nonlinearity 3 - Broad band $[0,1400] \mathrm{Hz}-N=5$ : Graph of $f \mapsto$ $\log _{10} h(2 \pi f)$ for the reference solution (black line), RML-solution (red line) and RMNL-solution (blue line). Horizontal axis frequency in Hertz. Vertical axis $f \mapsto \log _{10} h(2 \pi f)$. 


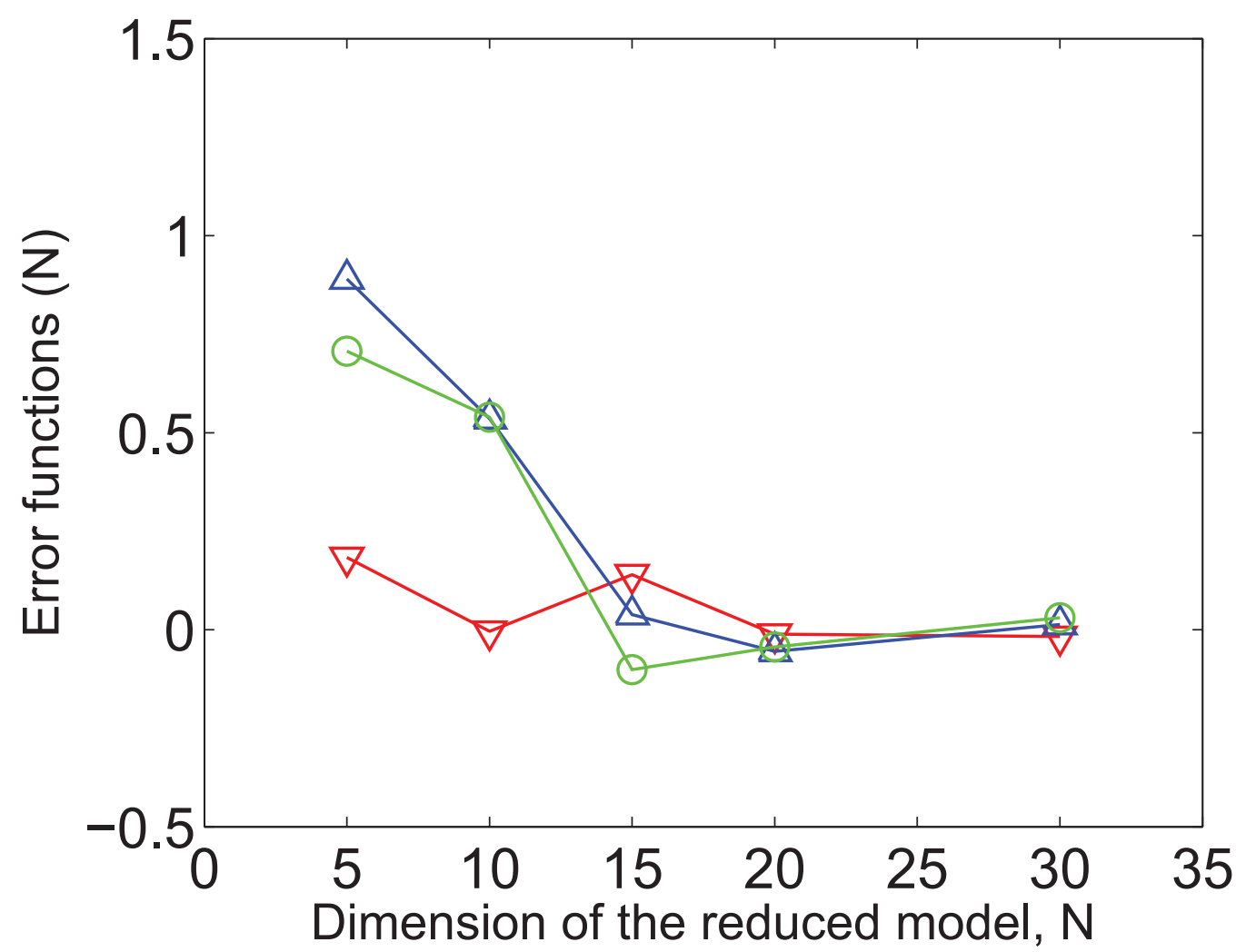

Figure 14: Nonlinearity 3 - Broad band [0, 1400] Hz - Efficiency of the LIN-solution and POD-solution in function of dimension $N$ of the reduced model. Graphs of the error functions versus $N$ : $e_{\text {REF-LIN }}(N)$ (triangle down), $e_{\text {REF-POD }}(N)$ (triangle up), $e_{\text {LIN-POD }}(N)$ (circle). 


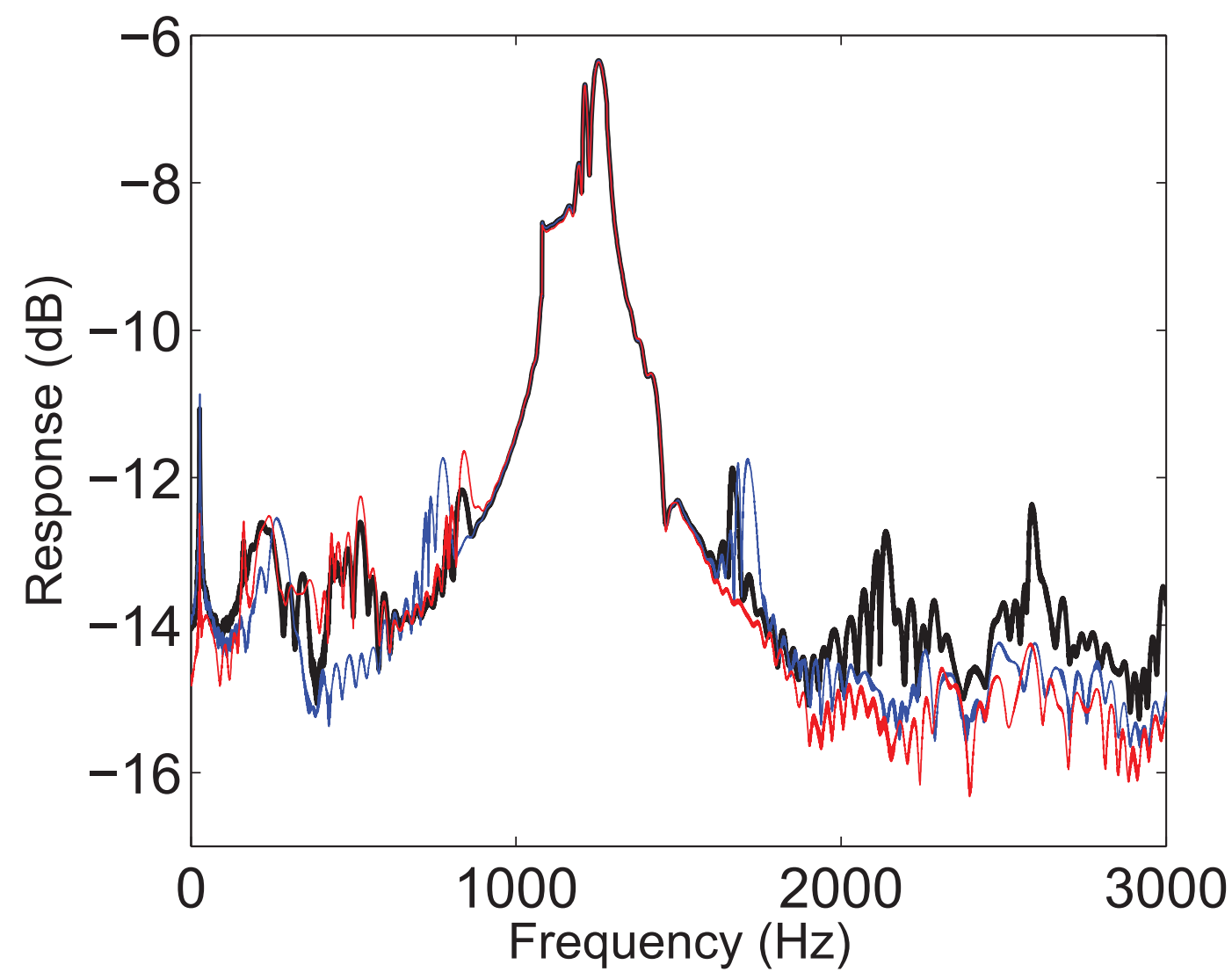

Figure 15: Nonlinearity 3 - Narrow band [1080,1280] $\mathrm{Hz}-N=5$ : Graph of $f \mapsto \log _{10} h(2 \pi f)$ for the reference solution (black line), RML-solution (red line) and RMNL-solution (blue line). Horizontal axis frequency in Hertz. Vertical axis $f \mapsto \log _{10} h(2 \pi f)$. 


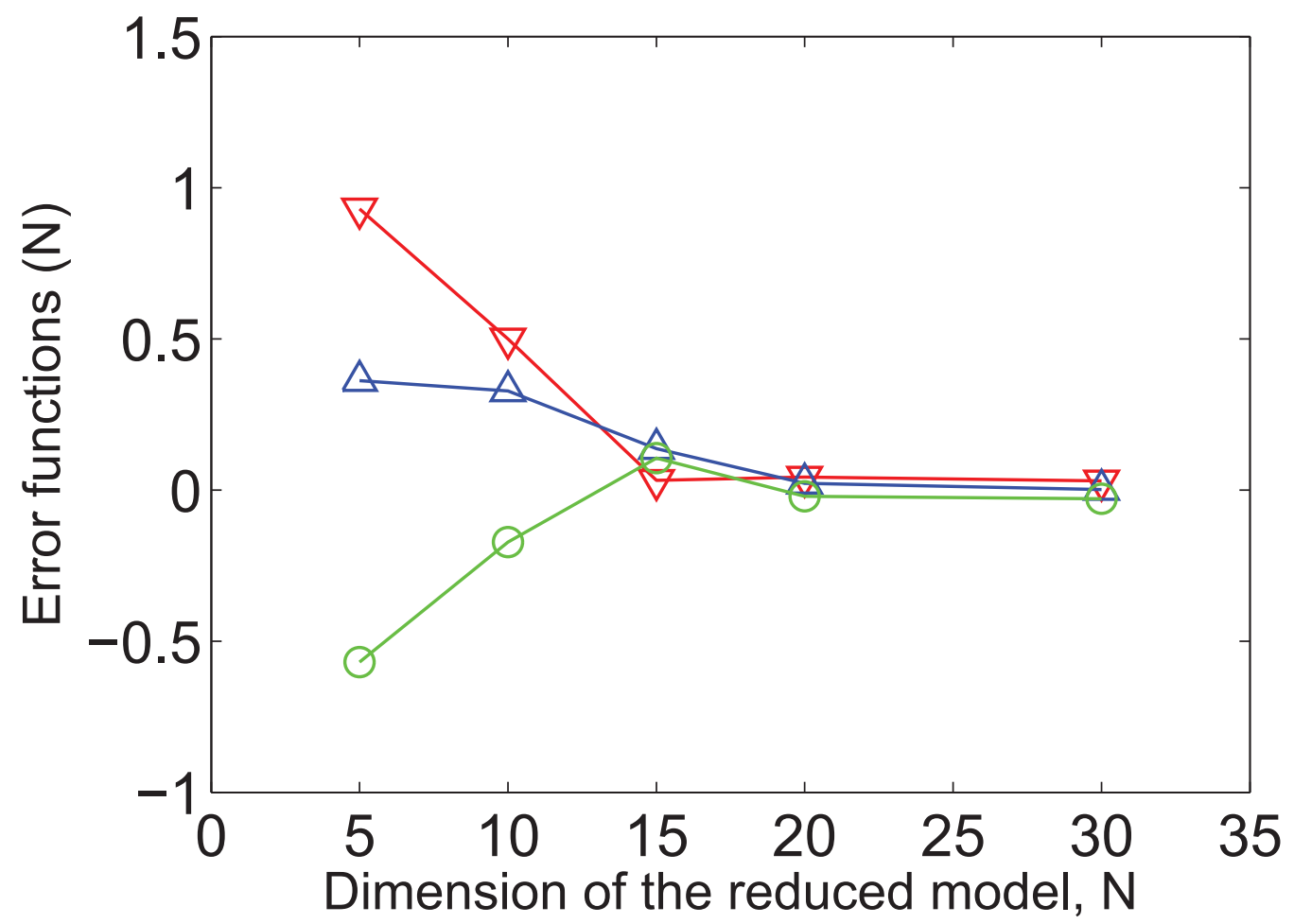

Figure 16: Nonlinearity 3 - Narrow band $[1080,1280] \mathrm{Hz}$ - Efficiency of the LINsolution and POD-solution in function of dimension $N$ of the reduced model. Graphs of the error functions versus $N: e_{\text {REF-LIN }}(N)$ (triangle down), $e_{\text {REF-POD }}(N)$ (triangle up), $e_{\mathrm{LIN}-\mathrm{POD}}(N)$ (circle). 